\title{
INTERPOLATING SEQUENCES IN POLYDISKS
}

\author{
BY
}

\author{
ERIC P. KRONSTADT $\left({ }^{1}\right)$
}

ABSTRACT. Let $D^{n}$ be the unit polydisk in $C^{n}, A$ be a uniform algebra, $H^{\infty}\left(D^{n}, A\right)$, the space of bounded analytic $A$ valued functions on $D^{n}$, $l^{\infty} A$, the space of bounded sequences of elements in $A$. A sequence, $S=$ $\left\{a_{i}\right\}_{i=1}^{\infty} \subset D^{n}$ will be called an interpolating sequence with respect to $A$ if the map $T: H^{\infty}\left(D^{n}, A\right) \rightarrow l^{\infty} A$ given by $T(f)=\left\{f\left(a_{i}\right)\right\}_{i=1}^{\infty}$ is surjective. In 1958, L. Carleson showed that for $n=1, S$ is interpolating w.r.t. C iff $S$ satisfies a certain zero-one interpolation property called uniform separation. We generalize this result to cases where $n>1$ and $A \neq C$. In particular, we show that if $S \subset D^{n}$ is uniformly separated and $s \subset w_{1} \times w_{2} \times \cdots \times w_{n}$ (where each $W_{j}$ is a region in $D$ lying between two circular arcs which intersect twice on the boundary of $D$ ) then $S$ is an interpolating sequence w.r.t. any uniform algebra. If $S \subset D^{n}$ is uniformly separated and $S \subset D \times w_{2} \times \cdots \times w_{n}$ then $S$ is interpolating w.r.t. C. Other examples and generalizations of interpolating sequences are discussed.

Let $A$ be a uniform algebra, i.e. a closed subalgebra of the continuous functions on a compact Hausdorff space which separates points, contains constants, and is equipped with the sup-norm topology. (See [2] and [7] for detailed descriptions of uniform algebras.) Let $D^{n}$ be the (open) unit polydisk in $\mathbf{C}^{n}$; let $H^{\infty}\left(D^{n}, A\right)$ be the Banach algebra of bounded, analytic, $A$-valued functions on $D^{n}$ equipped with the sup-norm (see [5, pp. 224-232] for a description of such functions); and let $l^{\infty} A$ be the Banach algebra of bounded sequences of elements of $A$. If $S=\left\{a_{i}\right\}_{i=1}^{\infty} \subset D^{n}$ is a sequence of points in $D^{n}$, we can define a map, $T: H^{\infty}\left(D^{n}, A\right) \rightarrow l^{\infty} A$ by $T(f)=\left\{f\left(a_{i}\right)\right\}_{i=1}^{\infty}$. We will say $S$ is an interpolating sequence w.r.t. $A$ if $T$ is surjective. If $S$ is an interpolating sequence w.r.t. C, we will simply say $S$ is an interpolating sequence, and if $S$ is an

Received by the editors May 23, 1973.

AMS (MOS) subject classifications (1970). Primary 30A80, 46J 10, 32E25; Secondary 30A96, 32A30, 46J15.

Key words and phrases. Interpolating sequence, uniformly separated sequence, general interpolating sequence, nontangential wedge, uniform algebra valued functions.

( ${ }^{1}$ ) The results presented in this paper represent part of the author's doctoral dissertation which was written at Harvard University under the direction of Professor Andrew M. Gleason. 
interpolating sequence w.r.t. every uniform algebra, we still say $S$ is a general interpolating sequence.

If $S=\left\{a_{i}\right\}_{i=1}^{\infty} \subset D^{n}$ is an interpolating sequence, then it is known that $S$ must be uniformly separated, i.e. there exists a constant $M$ and functions $f_{1}$, $f_{2}, \cdots \in H^{\infty}\left(D^{n}\right)$ such that for all $i,\left\|f_{i}\right\| \leqslant M$ and $f_{i}\left(a_{i}\right)=1$ while $f_{i}$ is zero on the remaining points of $S$. (We will use $H^{\infty}\left(D^{n}\right)$ for $H^{\infty}\left(D^{n}, \mathrm{C}\right)$.) In 1958, L. Carleson [3] showed that for $S \subset D\left(D=D^{1}\right)$, uniform separation is a necessary and sufficient condition that $S$ be an interpolating sequence (w.r.t. C). In this paper we attempt to extend Carleson's results to sequences in $D^{n}$ and to general interpolating sequences. We succeed to the extent of showing that uniform separation is sufficient in a large class of sequences in $D^{n}$ and $D$.

After introducing notation in $\S 1$, we review in $\S 2$ some of the concepts and results presented in [10]. $\S \S 3$ and 4 are devoted to a number of techniques for constructing general interpolating sequences. $\$ 5$ contains the main result of this paper demonstrating that uniformly separated sequences in "wedges" of $D^{n}$ are general interpolating sequences. $\S 6$ presents some miscellaneous examples of general interpolating sequences, and the last section indicates some directions in which all these results might be generalized.

1. Throughout this paper, $A$ will be a uniform algebra. We will let $m_{A}$ denote the maximal ideal space or spectrum of $A . m_{A}$ is of course a subset of $A^{*}$, the dual space of $A$, and as such can be equipped with the weak-* topology in which it is a compact Hausdorff space. We will identify elements of $A$ with their Gelfand transforms, so we may consider elements of $A$ as functions on $m_{A}$

It should be noted that $m_{A}$ is naturally supplied with two topologies as a subset of $A^{*}$-the weak-* topology and the norm topology. We will sometimes have occasion to use both, and it may also happen that subsets of $m_{A}$ are naturally associated with other topologies. Throughout this paper, if $Q \subset m_{A}$, we will consistently use the symbol $\mathrm{Cl}(Q)$ to represent the weak-* closure of $Q$ and will explicitly mention all other topologies. We will also use the symbol $\mathrm{Hk}(Q)$ for the hull of the kernel of $Q$, i.e.

$$
\operatorname{Hk}(Q)=\left\{s \in m_{A}: x(s)=0 \text { for every } x \in A \text { for which } x(Q)=0\right\} .
$$

The norm topology for $m_{A}$ is equivalent to the one induced by the "pseudo-hyperbolic" metric:

$$
\rho(z, w)=\operatorname{Sup}\{|x(z)|: x \in A,\|x\| \leqslant 1 \text { and } x(w)=0\} .
$$

We will use $D$ to represent the open unit disk in the complex plane, C, and $D^{n}$ for the open unit polydisk in $n$-dimensional complex space $\mathbf{C}^{n} \cdot T^{n}$ will refer to the essential boundary of $D^{n}$, i.e. 


$$
T^{n}=\left\{\left\langle z^{(1)}, z^{(2)}, \cdots, z^{(n)}\right\rangle \in \mathbf{C}^{n}:\left|z^{(j)}\right|=1 \text { for every } j, j=1, \cdots, n\right\} .
$$

$H^{\infty}(D)$ and $H^{\infty}\left(D^{n}\right)$ are, of course, the algebras of bounded analytic complex valued functions on $D$ and $D^{n}$ respectively. $H^{\infty}(D, A)$ and $H^{\infty}\left(D^{n}, A\right)$ will be the algebras of bounded analytic $A$-valued functions on $D$ and $D^{n}$ respectively.

$m_{1}, m_{n}$ will be written instead of $m_{H^{\infty}(D)}$ and $m_{H^{\infty}\left(D^{n}\right)}$ and points in $D$ (or $D^{n}$ ) will be identified with their associated point evaluation in $m_{1}$ (or $m_{n}$ ). Finally, we will often (but not always) use a subscript for the pseudohyperbolic metrics on $m_{n}$, e.g. $\rho_{1}$ will be the pseudo-hyperbolic metric on $m_{1}$. These metrics can be described for the cases where points are contained inside the polydisk involved, i.e. for $n=1, z, w \in D$, then $\rho_{1}(z, w)=$ $|(z-w) /(1-z \bar{w})|$. For $n>1$

$$
\left\langle z^{(1)}, z^{(2)}, \cdots, z^{(n)}\right\rangle,\left\langle w^{(1)}, \cdots, w^{(n)}\right\rangle \in D^{n} ;
$$

then

$$
\begin{aligned}
\rho_{n}\left(\left\langle z^{(1)}, \cdots, z^{(n)}\right\rangle,\left\langle w^{(1)}, \cdots, w^{(n)}\right\rangle\right) \\
\quad=\operatorname{Max}\left\{\rho_{1}\left(z^{(j)}, w^{(j)}\right): j=1,2, \cdots, n\right\} .
\end{aligned}
$$

In other words, the pseudo-hyperbolic distance between two points in $D^{n}$ is the maximum of the pseudo-hyperbolic distances of the projections of the points on each of the coordinate axes.

2. General interpolating, uniformly separated, and $\alpha$-separated sequences. If $S$ is an interpolating sequence w.r.t. $A$, the map $T: H^{\infty}\left(D^{n}, A\right) \rightarrow l^{\infty} A$ described above is surjective, and the open mapping theorem implies the existence of a constant $M_{A}$ such that for any element $\left\{X_{i}\right\}_{i=1}^{\infty} \in l^{\infty} A$, there exists $f \in H^{\infty}\left(D^{n}, A\right)$ such that $\|f\| \leqslant M_{A}\left(\operatorname{Sup}_{i}\left\|X_{i}\right\|\right)$ and $f\left(a_{i}\right)=X_{i}, i=1,2, \cdots$. Any such $M_{A}$ will be called an interpolating bound for $S$ w.r.t. $A$.

PROPOSITION 2.1. If $S$ is a general interpolating sequence, there exists a single constant $M$ with the property that if $A$ is a uniform algebra, there is an interpolating bound for $S$ w.r.t. $A$ which is less than $M$.

Proof. For any uniform algebra $A$, let $N_{A}=\operatorname{Inf}\{M: M$ is an interpolating bound for $S$ w.r.t. $A$ \}. Suppose the theorem is false, then we can choose uniform algebras $A_{1}, A_{2}, \cdots$ so that $N_{A_{k}} \geqslant k$ for $k=1,2, \cdots$. Consider the Cartesian product $Q=A_{1} \times A_{2} \times A_{3} \times \cdots$, and the algebra $A=$ $\left\{x=\left\langle x_{1}, x_{2}, \cdots\right\rangle \in Q: \operatorname{Sup}_{i}\left\|x_{i}\right\|_{A_{i}}<\infty\right\}$. $A$ will be a Banach algebra under coordinate-wise addition and multiplication and the norm given by $\|x\|=$ $\left\|\left\langle x_{1}, x_{2}, x_{3}, \cdots\right\rangle\right\|=\operatorname{Sup}_{i}\left\{\left\|x_{i}\right\|_{A_{i}}\right\}$. Since $A_{i}$ is a uniform algebra $\left\|x_{i}^{2}\right\|_{A_{i}}=$ $\left(\left\|x_{i}\right\|_{A_{i}}\right)^{2}$ for all $x_{i} \in A_{i}$, hence $\left\|x^{2}\right\|=\|x\|^{2}$ for all $x \in A$, and consequently 
the Gelfand transform of $A$ is an isometric isomorphism, so $A$ is isomorphic to a uniform algebra. We note that the projection maps $\pi_{j}: A \rightarrow A_{j}$ are all linear, of norm one. Let $M$ be a general interpolating bound for $S$ with respect to $A$. Fix $k>M$, and consider $y_{1}, y_{2}, \cdots \in A_{k}$ such that $\left\|y_{i}\right\|_{A_{k}} \leqslant$ 1. Define $t_{j} \in A$ by $t_{j}=\left\langle 0,0, \cdots, 0, y_{j}, 0, \cdots\right\rangle$ (where the $y_{j}$ is in the $k$ th place); then $\left\|t_{j}\right\| \leqslant 1$ for all $j$. Hence there is a bounded analytic function $f: D^{n} \rightarrow A$ such that $f\left(a_{j}\right)=t_{j}$ for $j=1,2, \cdots$ and $\|f(z)\| \leqslant M$ for all $z \in D^{n}$. Then $g=\pi_{k} \circ f$ will be a bounded analytic function, $g: D^{n} \rightarrow A_{k}$ such that $g\left(a_{j}\right)=y_{j}$ for $j=1,2, \cdots$, and $\|g(z)\|_{A_{k}} \leqslant M$ for all $z \in D^{n}$. Therefore $N_{A_{k}} \leqslant M$, which contradicts $k \leqslant N_{A_{k}}$ and $M<k$, so that the $A_{k}$ 's must not have existed in the first place.

Definition 2.2. Any constant $M$ with the property of Proposition 1.1 will be called a general interpolating bound for $S$.

DEFInItion 2.3. A sequence $S=\left\{a_{i}\right\}_{i=1}^{\infty} \subset D^{n}$ will be called a uniformly separated (u.s.) sequence if there exists a constant $M>0$ and functions $f_{1}$, $f_{2}, \cdots \in H^{\infty}\left(D^{n}\right)$ with the property that $\left\|f_{i}\right\| \leqslant M$ for all $i$, and $f_{i}\left(a_{i}\right)=1$ while $f_{i}\left(a_{j}\right)=0$ for $i \neq j, i=1,2, \cdots$. If $S$ is u.s., the constant $M$ will be called a u.s. bound for $S$.

We have noted in the introduction that if $S \subset D$, then $S$ is interpolating iff $S$ is u.s. In this case, by means of Blaschke products, we see that $S$ is a u.s. sequence in $D$ iff

$$
\operatorname{Inf}_{i} \prod_{j=1 ; j \neq 1}^{\infty}\left|\frac{a_{i}-a_{j}}{1-a_{i} \vec{a}_{j}}\right|>0
$$

This last condition is equivalent to the following property: There exist constants $M>0$ and $p>0$ and functions $\theta_{1}, \theta_{2}, \cdots \in H^{\infty}(D)$ such that

(1) $\left\|\theta_{i}\right\| \leqslant 1$ for all $i$.

(2) $\theta_{i}\left(a_{i}\right)=0$ while $\left|\theta_{i}\left(a_{j}\right)\right|>p$ if $i \neq j$.

(3) $\sum_{j=1}^{\infty} 1-\left|\theta_{j}\left(a_{i}\right)\right| \leqslant M$ for all $i$.

(4) $\sum_{j=1}^{\infty}\left|1-\theta_{j}(z)\right|$ converges uniformly on compact subsets of $D$. (The function $\theta_{j}(z)$ is just $\bar{a}_{j} /\left|a_{j}\right| \cdot\left(a_{j}-z\right) /\left(1-\bar{a}_{j} z\right)$.) We define a similar, stronger, property which is sufficient for general interpolation.

DEFINITION 2.4. A sequence $S=\left\{a_{i}\right\}_{i=1}^{\infty} \subset D^{n}$ will be called $\alpha$-separated if there exist constants $m, p>0$ and functions $\alpha_{1}, \alpha_{2}, \alpha_{3}, \cdots \in H^{\infty}\left(D^{n}\right)$ such that

(1) $\left\|\alpha_{i}\right\| \leqslant 1$ for all $i$.

(2) $\alpha_{i}\left(a_{i}\right)=0$ while $\left|\alpha_{i}\left(a_{j}\right)\right|>p$ for $i \neq j$.

(3) $\sum_{j=1}^{\infty}\left|1-\alpha_{j}\left(a_{i}\right)\right| \leqslant m$ for all $i$.

(4) $\sum_{j=1}^{\infty}\left|1-\alpha_{j}(z)\right|$ converges uniformly on compact subsets of $D^{n}$. 
Note. Definition 2.4 includes sequences in polydisks, and the four conditions differ from the four listed above only in the placement of the absolute value signs in (3).

THEOREM 2.5. If $S=\left\{a_{i}\right\}_{i=1}^{\infty} \subset D^{n}$ is $\alpha$-separated, then $S$ is a general interpolating sequence with bound depending only on the parameters $m, p$ of the definition of $\alpha$-separation.

Proof. This is Theorem 2.2 of [10].

3. Jiggling theorems. We would like to apply Theorem 2.5 to a fairly general class of sequences in $D^{n}$. In order to do this, we will have to develop some techniques for constructing new interpolating sequences from old ones. The first of these techniques involves "jiggling" the points of a known interpolating sequence. The other techniques include taking unions and Cartesian products of two or more sequences. The jiggling theorems provide a small neighborhood around each point of an interpolating sequence in which that point is free to move without affecting the interpolating property. They are based on the following two elementary lemmas.

Lemma 3.1. Suppose $A, B$ are two Banach spaces and $T \in \operatorname{Hom}(A, B)$ and $E \subset B$ is the unit ball of $B$. Suppose there exist constants $0<k<1$ and $K>0$ such that for all $Y \in E$, there is an $X \in A$ such that $\|T X-Y\|$ $\leqslant k$ and $\|X\| \leqslant K$, then $T$ is onto, and for all $Y$ in $B$ there exists $X$ in $A$ such that $T X=Y$ and $\|X\| \leqslant K /(1-k)$.

Proof. See for example Theorem 1.2 of [1] for a generalization.

LEMma 3.2. If $f: D^{n} \rightarrow A$ is analytic, $\|f(z)\| \leqslant 1$ for all $z \in D^{n}$, then for $z, w \in D^{n},\|f(z)-f(w)\| \leqslant 2 \rho(z, w)$.

Proof. Let $z, w \in D^{n}$ be fixed, and choose $\phi \in A^{*}$ ( $A^{*}$ is the dual space of $A$ ) such that $\|\phi\|=1$ and $\phi(f(z)-f(w))=\|f(z)-f(w)\|$. Let $F=$ $\phi \circ f$, then $F \in H^{\infty}\left(D^{n}\right)$ and $\|F\| \leqslant 1$, so

$$
\left|\frac{F(z)-F(w)}{1-F(z) \overline{F(w)}}\right| \leqslant \rho(z, w)
$$

hence $|F(z)-F(w)| \leqslant 2 \rho(z, w)$. Consequently $\|f(z)-f(w)\| \leqslant 2 \rho(z, w)$.

THEOREM 3.3. If $S=\left\{a_{i}\right\}_{i=1}^{\infty} \subset D^{n}$ is an interpolating sequence w.r.t. $A$ with interpolating bound, $M_{A}$ (w.r.t. $A$ ), and $\epsilon \leqslant 1 / 4 M_{A}$, and if we choose $b_{i} \in D^{n}$ such that $\rho_{n}\left(a_{i}, b_{i}\right) \leqslant \epsilon$ for all $i, i=1,2, \cdots$ then $T=\left\{b_{i}\right\}_{i=1}^{\infty}$ is an interpolating sequence with bound $2 M_{A}$ (w.r.t. $A$ ). 
Proof. Let $E$ be the unit ball of $l^{\infty} A$. If $\left\{X_{i}\right\}_{i=1}^{\infty} \in E$ and $f: D^{n} \rightarrow$ $A$ is an analytic function with $f\left(a_{i}\right)=X_{i}$ and $\|f\| \leqslant M_{A}$; then

$$
\left\|f\left(b_{i}\right)-X_{i}\right\|=\left\|f\left(b_{i}\right)-f\left(a_{i}\right)\right\| \leqslant 2 M_{A} \rho_{n}\left(a_{i}, b_{i}\right) \leqslant 1 / 2 .
$$

Hence Lemma 3.1 applies and $T$ is an interpolating sequence w.r.t. $A$ with bound $2 M_{A}$.

COROLLARY 3.4 (JIGGLING THEOREM FOR GENERAL INTERPOLATING SEQUENCES). If $S=\left\{a_{i}\right\}_{i=1}^{\infty} D^{n}$ is a general interpolating sequence with general interpolating bound $M$, and $\epsilon=1 / 4 M$, and if we choose $b_{i} \in D^{n}$ such that $\rho_{n}\left(a_{i}, b_{i}\right) \leqslant \epsilon$ for $i=1,2, \cdots$, then $T=\left\{b_{i}\right\}_{i=1}^{\infty}$ is a general interpolating sequence with bound $2 M$.

The jiggling theorems tell us that a sequence which is "close" to a given interpolating sequence is also interpolating. We can attempt an analogous characterization of u.s. sequences. We remark that although we generally take the terms "interpolating" and "uniformly separated" to apply to infinite sequences, they can also be applied to finite sets of points. Shapiro and Shields [14] showed that a u.s. sequence (finite or infinite) in $D$ with u.s. bound $1 / \delta$ is interpolating, with interpolating bound $\left(2 / \delta^{5}\right)(1-2 \log \delta)$ or, put another way, if $S \subset D$ is a u.s. sequence with u.s. bound $1 / \delta$ and $h$ is a bounded function on $S$, $\|h\|_{\text {Sup }} \leqslant \delta^{5} / 2(1-2 \log \delta)$, then there exists $f \in H^{\infty}(D)$ such that $f \nmid S=h$ and $\|f\| \leqslant 1$. We will use the last estimate frequently, so in order to simplify the notation we will write

$$
\kappa(\delta)=\delta^{5} / 2(1-2 \log \delta) .
$$

The following theorem shows that if a sequence in $D$ is "almost" uniformly separated, then it is indeed u.s.

THEOREM 3.5. Let $S=\left\{a_{i}\right\}_{i=1}^{\infty} \subset D$ and suppose there exists a constant $m, 0<m<1$ and functions $f_{1}, f_{2}, \cdots \in H^{\infty}(D)$ such that

$$
\left\|f_{i}\right\| \leqslant 1 \quad \text { for } i=1,2, \cdots ; \quad\left|f_{i}\left(a_{i}\right)\right|>m \quad \text { for } i=1,2, \cdots ;
$$

and

$$
\left|f_{i}\left(a_{j}\right)\right| \leqslant \epsilon_{0}=(m / 2) \kappa(m / 2) \text { for } i \neq j .
$$

Then $S$ is uniformly separated (hence interpolating) and $S$ has u.s. bound $2 / m$.

Proof. For $N=2,3, \cdots$ we show that any set $T$ of $N$ distinct points of $S$ is uniformly separated with u.s. bound $2 / \mathrm{m}$. The theorem then follows from standard pointwise limit arguments for $H^{\infty}(D)$. 
We proceed by induction. If $N=2$, let $z, w$ be two distinct points in $S$. The hypothesis of the theorem guarantees the existence of $f \in H^{\infty}(D),\|f\| \leqslant$ $1,|f(z)|>m$ while $|f(w)|<\epsilon_{0}<m / 2$. Hence

$$
\rho(z, w) \geqslant\left|\frac{f(z)-f(w)}{1-f(z) \bar{f}(w)}\right| \geqslant|f(z)|-|f(w)| \geqslant m-\epsilon_{0}>m / 2,
$$

so our assertion is true for sets of two points.

Suppose we have demonstrated our assertion for sets of $N-1$ or fewer points of $S$. Let $T$ consist of $N$ distinct points of $S$ and let $z_{0} \in T$. Let $Q=T \backslash\left\{z_{0}\right\}$. The hypothesis of the theorem guarantees the existence of $f \in$ $H^{\infty}(D)$ such that $\|f\| \leqslant 1,|f(w)|<\epsilon_{0}$ for all $w \in Q$, and $\left|f\left(z_{0}\right)\right|>m$. Define $F: Q \rightarrow \mathrm{C}$ by

$$
F(w)=f(w)\left(1-w \overline{z_{0}}\right) /\left(w-z_{0}\right) \text { for } w \in Q ;
$$

then for all $w \in Q,|F(w)| \leqslant 2 \epsilon_{0} / m$ (since $\left|\left(1-w \overline{z_{0}}\right) /\left(w-z_{0}\right)\right|=1 / \rho\left(z_{0}, w\right)$, we can apply the case $N=2$ ).

By the induction hypothesis, since $Q$ has $N-1$ points, $Q$ is u.s. with u.s. bound $2 / m$. Consequently we can apply the Shapiro and Shields result to obtain $h \in H^{\infty}(D)$ such that $\|h\| \leqslant 1$ and $h \uparrow Q=F$. Define $g \in H^{\infty}(D)$ by

$$
g(z)=\frac{1}{2}\left(f(z)-\frac{z-z_{0}}{1-z \bar{z}_{0}} h(z)\right) .
$$

Then $\|g\| \leqslant 1, g(w)=0$ for all $w \in Q=T \backslash\left\{z_{0}\right\}$ and $\left|g\left(z_{0}\right)\right|>m / 2$. This argument will produce such a function for any point in $T$, hence $T$ is u.s. with bound $2 / \mathrm{m}$. The induction argument and thus the proof of Theorem 3.5 is complete.

We remark that a theorem similar to Theorem 3.5 is always available whenever uniform separation is necessary and sufficient for interpolation and an explicit expression for the interpolating bound of a sequence, given its u.s. bound, is available.

For sequences in polydisks, Theorem 3.5 has the following consequences.

Let $a=\left\langle a^{(1)}, a^{(2)}, \cdots, a^{(n)}\right\rangle \in D^{n}$ and let $r<1$; consider the region $D_{k}(a, r)=\left\{z=\left\langle z^{(1)}, \cdots, z^{(n)}\right\rangle: \rho_{1}\left(z^{(j)}, a^{(j)}\right)<r\right.$ for all $j=1,2, \cdots, n$ except $j=k$ \}. $D_{k}(a, r)$ might be called a "narrow polydisk" in the sense that all but one of its polydisk radii are small. $D_{k}(a, r)$ contains a copy of $D$ obtained by the embedding

$$
\sigma: z \rightarrow\left\langle a^{(1)}, \cdots, a^{(k-1)}, z, a^{(k+1)}, \cdots, a^{(n)}\right\rangle,
$$

and $D_{k}(a, r)$ consists of points in $D^{n}$ whose pseudo-hyperbolic distances from this disk are less than $r$. Theorem 3.5 implies that a u.s. sequence contained 
in a suitable narrow polydisk is interpolating.

Corollary 3.6. Suppose $S=\left\{a_{i}\right\}_{i=1}^{\infty} \subset D^{n}$ is a u.s. sequence with u.s. bound $M$, and suppose there exists $a \in D^{n}$ such that $S \subset D_{k}\left(a, \epsilon_{0}\right)$ where $\epsilon_{0}=(1 / 4 M) \kappa(1 / 4 M)$. Let $\pi_{j}: D^{n} \rightarrow D$ be the projection onto the $j$ th coordinate axis for $j=1,2, \cdots, n$. Then $\pi_{k}(S)$ is u.s. in $D$ with u.s. bound $4 M$ and consequently $S$ is interpolating.

Proof. Let $\sigma: z \rightarrow\left\langle a^{(1)}, \cdots, a^{(k-1)}, z, a^{(k+1)}, \cdots, a^{(n)}\right\rangle$ and let $\tau=\sigma \circ \pi_{k}$ so that $\tau: D^{n} \rightarrow \sigma(D)$. Clearly, if $z \in D_{k}\left(a, \epsilon_{0}\right)$ then $\rho_{n}(z, \tau(z))<\epsilon_{0}$.

$S$ uniformly separated with bound $M$ means that there exist functions $f_{1}, f_{2}, \cdots, \in H^{\infty}\left(D^{n}\right)$ such that $\left\|f_{i}\right\| \leqslant 1$ for all $i$ and

$$
f_{i}\left(a_{j}\right)= \begin{cases}0 & \text { if } i \neq j \\ 1 / M & \text { if } i=j\end{cases}
$$

Then since $\rho_{n}\left(a_{i}, \tau\left(a_{i}\right)\right)<\epsilon_{0}<1 / 2 M$ for all $i,\left|f_{i}\left(\tau\left(a_{i}\right)\right)\right|>1 / 2 M$ for all $i$, and $\left|f_{i}\left(\tau\left(a_{j}\right)\right)\right|<$ $\epsilon_{0}=(1 / 4 M) \kappa(1 / 4 M)$ for all $i \neq j$. So, if $g_{i}=f_{i} \circ \sigma, g_{i} \in H^{\infty}(D),\left\|g_{i}\right\| \leqslant 1$ and $\left|g_{i}\left(\pi_{k}\left(a_{i}\right)\right)\right|>1 / 2 M$ for all $i$ and $\left|g_{i}\left(\pi_{k}\left(a_{j}\right)\right)\right|<(1 / 4 M) \kappa(1 / 4 M)$ for all $i \neq j$.

Therefore, $\pi_{k}(S)=\left\{\pi_{k}\left(a_{i}\right)\right\}_{i=1}^{\infty} \subset D$ satisfies the hypothesis of Theorem 3.5 with $m=1 / 2 M$ and is consequently u.s. with bound $4 M$. Finally, $\pi_{k}(S)$, uniformly separated in $D$, implies $\pi_{k}(S)$ is interpolating, so if $\left\langle\lambda_{1}, \lambda_{2}, \cdots\right\rangle \in l^{\infty}$, there exists $h \in H^{\infty}(D)$ such that $\left(h \circ \pi_{k}\right)\left(a_{i}\right)=h\left(\pi_{k}\left(a_{i}\right)\right)=\lambda_{i}$. Clearly $h \circ \pi_{k} \in$ $H^{\infty}\left(D^{n}\right)$, so $S$ is interpolating.

4. Cartesian products and unions. Taking Cartesian products of general interpolating sequences preserves the general interpolating property because $H^{\infty}\left(D^{n}, A\right)$ is itself isomorphic to a uniform algebra. (Obviously $H^{\infty}\left(D^{n}, A\right)$ is a Banach algebra and it is clear that if $f \in H^{\infty}\left(D^{n}, A\right),\left\|f^{2}\right\|=\|f\|^{2}$ since $A$ is a uniform algebra. Therefore, the Gelfand mapping for $H^{\infty}\left(D^{n}, A\right)$ is an isometric isomorphism, and $H^{\infty}\left(D^{n}, A\right)$ is isomorphic to a uniform algebra.)

Proposition 4.1. If $S=\left\{a_{i}\right\}_{i=1}^{\infty} \subset D^{n}$ is a general interpolating sequence with bound $M$, then $S \times S \subset D^{2 n}$ is a general interpolating sequence with bound $M^{2}$.

Proof. Let $A$ be any uniform algebra.

If $\left\{X_{i j}\right\}_{i=1 ; j=1}^{\infty} \subset A$ then for each $i$, there is $f_{i} \in H^{\infty}\left(D^{n}, A\right)$ such that $f_{i}\left(a_{j}\right)=X_{i j}$ where $\left\|f_{i}\right\| \leqslant M \sup _{j}\left\|X_{i j}\right\|$. But we can interpolate into $H^{\infty}\left(D^{n}, A\right)$ i.e. there exists $F: D^{n} \rightarrow H^{\infty}\left(D^{n}, A\right)$ such that $F$ is bounded and analytic, $F\left(a_{i}\right)=f_{i}$ and

$$
\|F(z)\| \leqslant M \operatorname{Sup}_{i}\left\|f_{i}\right\| \leqslant M^{2} \sup _{i, j}\left\|X_{i j}\right\| \quad \text { for each } z \in D^{n} .
$$


Let $G: D^{2 n} \rightarrow A$ be defined by $G(\langle z, w\rangle)=(F(z))(w)$; then $G$ will be analytic, $\|G(\langle z, w\rangle)\| \leqslant M^{2} \sup _{i, j}\left\|X_{i j}\right\|$ for all $\langle z, w\rangle \in D^{n} \times D^{n}$ and

$$
G\left(\left\langle a_{i}, a_{j}\right\rangle\right)=\left(F\left(a_{i}\right)\right)\left(a_{j}\right)=f_{i}\left(a_{j}\right)=X_{i j} .
$$

The above arguments also show that if $S_{1}, S_{2}, \cdots \subset D^{n}$ are interpolating sequences (w.r.t. $A$ ) each with bound $M_{1}$ and $T_{1}, T_{2}, T_{3}, \cdots \subset D^{n}$ are generally interpolating sequences, each with bound $M_{2}$, and $S=\left\{a_{i}\right\}_{i=1}^{\infty} \subset D^{k}$ is a general interpolating sequence with bound $M_{0}$, then the sequence $\left\{\left\{a_{i}\right\} \times S_{i}\right\}_{i=1}^{\infty} \subset D^{n+k}$ is interpolating w.r.t. $A$ with bound $M_{1} M_{0}$, and the sequence $\left\{\left\{a_{i}\right\} \times T_{i}\right\}_{i=1}^{\infty} \subset D^{n+k}$ is a general interpolating sequence with bound $M_{2} M_{0}$.

In order to take the union of two interpolating sequences, we simply need a function which vanishes on one sequence and is a nonzero constant on the other. (See the work of Varopoulos [16] for other results on the unions of interpolating sets.)

Proposition 4.2. If $A$ is a uniform algebra, and $Q$ and $R$ are any two subsets in $m_{A}, \mathrm{Hk}(Q) \cap \mathrm{Hk}(R)=\varnothing$ implies the existence of elements $x$ and $y$ in $A$ such that

$$
x(Q)=0, \quad x(R)=1, \quad \text { and } \quad y(Q)=1, \quad y(R)=0 .
$$

Proof. Let $I_{Q}$ be the ideal (kernel) generated by $Q$, i.e. $I_{Q}=\{x \in$ $A: x(Q)=0\}$, similarly define $I_{R}$, and let $I$ be the ideal generated by $I_{Q} U$ $I_{R}$. If there is a maximal ideal $m \in m_{A}$ containing $I$ then $x(m)=0$ for all $x \in I_{Q} \cup I_{R}$. Hence $m \in \mathrm{Hk}(Q) \cap \mathrm{Hk}(R)$ which is impossible. Therefore, $I=A$ and there must exist $x \in I_{Q}$ and $y \in I_{R}$ such that $x+y=1$. Clearly $x$ and $y$ are the desired functions.

In the following theorem and corollaries we re-emphasize that the symbol $\mathrm{Cl}$ represents the weak-* closure in the maximal ideal space.

THEOREM 4.3. If $S \subset D^{n}$ is an interpolating sequence (w.r.t. C) then $\mathrm{Cl}(S)=\mathrm{Hk}(S)$.

Proof. Clearly $\mathrm{Cl}(S) \subset \mathrm{Hk}(S)$. Suppose $z \notin \mathrm{Cl}(S)$. Then there exists a weak-* neighborhood $N$ of $z$ which is disjoint from $\mathrm{Cl}(S)$. $N$ will contain a basic neighborhood of the form $\left\{w \in m_{n}:\left|f_{i}(w)\right|<\epsilon\right.$ where $f_{i} \in H^{\infty}\left(D^{n}\right)$, $f_{i}(z)=0$ for $\left.i=1,2, \cdots, k\right\}$. Hence $\mathrm{Cl}(S)=\bigcup_{i=1}^{k} S_{i}$ where $S_{i}=\mathrm{Cl}(S) \cap$ $\left\{w:\left|f_{i}(w)\right| \geqslant \epsilon / 2\right\}$. For each $i, i=1,2, \cdots, k, 1 / f_{i}(S)$ will be a bounded continuous function on the closed subset $S_{i}$ of the compact Hausdorf space $\mathrm{Cl}(S)$, so $1 / f_{i}$ has a bounded continuous extension to all of $\mathrm{Cl}(S)$; call this extension $h_{i}$. $S$ an interpolating sequence implies the existence of $g_{i} \in H^{\infty}\left(D^{n}\right)$ where 
$g_{i} \uparrow S=h_{i}$, in particular, $g_{i} \uparrow S_{i}=1 / f_{i}$. Therefore, $1-g_{i} f_{i} \in H^{\infty}\left(D^{n}\right)$ and has values 1 at $z$ and 0 on $S_{i}$. Finally let $f=\Pi_{i=1}^{k}\left(1-g_{i} f_{i}\right), f \in H^{\infty}\left(D^{n}\right)$, $f(z)=1$, and $f(S)=0$, so $z \notin \mathrm{Hk}(S)$.

COROLlaRY 4.4. If $S$ and $T$ are (general) interpolating sequences in $D^{n}$ with disjoint weak-* closures in $m_{n}$, then $S \cup T$ is a (general) interpolating sequence.

Proof. Suppose $S$ and $T$ are interpolating w.r.t. $A$. By Theorem 4.3, $\mathrm{Hk}(S) \cap \mathrm{Hk}(T)=\varnothing$. Hence by Proposition 4.2 there exist $f, g \in H^{\infty}\left(D^{n}\right)$ such that $f(S)=0, f(T)=1, g(S)=1$, and $g(T)=0$. Suppose we want to interpolate $X_{i}$ at each point $a_{i}$ of $S$ and $Y_{i}$ at each point $b_{i}$ of $T$, where $\left\{X_{i}\right\}_{i=1}^{\infty},\left\{Y_{i}\right\}_{i=1}^{\infty} \in l^{\infty} A$.

We know there exists $h_{1}, h_{2} \in H^{\infty}\left(D^{n}, A\right)$ such that $h_{1}\left(a_{i}\right)=X_{i}$ and $h_{2}\left(b_{i}\right)=Y_{i}$. So let $h=g h_{1}+f h_{2} . h \in H^{\infty}\left(D^{n}, A\right)$ and $h\left(a_{i}\right)=X_{i}$ and $h\left(b_{i}\right)=Y_{i}$ for all $i$.

We put the above results in a framework which we will find most useful.

Proposition 4.5. Suppose $m_{n}=G_{1} \cup G_{2} \cup \cdots \cup G_{m}$ where each $G_{i}$ is a weak.* open subset of $m_{n}$, and suppose $S \subset D^{n}$ is a discrete sequence such that $S \cap G_{i}$ is an interpolating sequence w.r.t. $A$ for $i=1,2, \cdots, m$. Then $S$ is an interpolating sequence w.r.t. A.

Proof. We recall that interpolating w.r.t. $A$ automatically implies interpolating w.r.t. C. Also we remark that if $Q$ is an interpolating sequence (w.r.t. C) in $D^{n}$, and $T \subset Q$, then there exists $f \in H^{\infty}\left(D^{n}\right)$ such that $f(T)=0$ and $f(Q \backslash T)=1$, hence $\mathrm{Cl}(T) \cap \mathrm{Cl}(Q \backslash T)=\varnothing$.

Let $S_{k}=S \cap\left(G_{1} \cup G_{2} \cup \cdots \cup G_{k}\right)$ we show $S_{k}$ is interpolating w.r.t. $A$ for $k=1,2, \cdots, n$. By hypothesis, $S_{1}$ is interpolating. Suppose $S_{k-1}$ is interpolating w.r.t. $A$. Let $G=G_{1} \cup G_{2} \cup \cdots \cup G_{k-1}$ so that $S_{k-1}=$ $S \cap G$ and let $T=S \cap\left(G_{k} \backslash G\right)$, then $S_{k-1}$ and $T$ will be disjoint interpolating sequences w.r.t. $A$. We show $\mathrm{Cl}\left(S_{k-1}\right) \cap \mathrm{Cl}(T)=\varnothing$ in which case Corollary 4.4 will imply $S_{k}=S_{k-1} \cup T$ is an interpolating sequence w.r.t. $A$.

For any $j,\left(S_{k-1} \cap G_{j}\right) \cup\left(T \cap G_{j}\right) \subset S \cap G_{j}$ and by hypothesis $\left(S_{k-1} \cap G_{j}\right) \cup\left(T \cap G_{j}\right)$ is interpolating, so by the remark at the beginning of this proof $\mathrm{Cl}\left(S_{k-1} \cap G_{j}\right) \cap \mathrm{Cl}\left(T \cap G_{j}\right)=\varnothing$. Now suppose $a \in \mathrm{Cl}\left(S_{k-1}\right) \cap$ $\mathrm{Cl}(T)$, then $a \in m_{n}$ implies $a \in G_{j}$ for some $j$ and since $G_{j}$ is an open set, $a \in \mathrm{Cl}\left(S_{k-1} \cap G_{j}\right) \cap \mathrm{Cl}\left(T \cap G_{j}\right)=\varnothing$. Hence $\mathrm{Cl}\left(S_{k-1}\right) \cap \mathrm{Cl}(T)=\varnothing$ as desired.

We remark that the above proposition is clearly true if we replace the phrase "interpolating w.r.t. $A$ " with "general interpolating". Finally, we give a set of concrete conditions under which we will be able to apply Proposition 4.5. 
LEMma 4.6. Suppose we are given $F_{1}, F_{2}, \cdots, F_{k}$ where each $F_{j}$ is a finite collection of functions in $H^{\infty}\left(D^{n}\right)$. Fix two positive constants $0<r<t$ and let $F_{j}=\left\{z \in D^{n}:|f(z)| \leqslant r\right.$ for all $\left.f \in F_{j}\right\}$ and $E_{j}=\left\{z \in D^{n}:|f(z)|<t\right.$ for all $f \in F_{j}$ \}. Then there exists $G_{0}, G_{1}, \cdots, G_{k}$ open subsets of $m_{n}$ such that

(1) $G_{0} \cap D^{n}=D^{n} \backslash\left(\bigcup_{j=1}^{k} F_{j}\right)$ and $G_{j} \cap D^{n}=E_{j}$ for $j=1,2, \cdots, k$,

(2) $m_{n}=\bigcup_{j=0}^{k} G_{j}$.

Proof. Let

$$
G_{0}=m_{n} \backslash \mathrm{Cl}\left(\bigcup_{j=1}^{k} F_{j}\right), \quad G_{j}=m_{n} \backslash \mathrm{Cl}\left(D^{n} \backslash E_{j}\right) \quad \text { for } j=1,2, \cdots, k \text {; }
$$

then clearly $G_{0} \cap D^{n}=D^{n} \backslash\left(\bigcup_{j=1}^{k} F_{j}\right)$ and $G_{j} \cap D^{n}=E_{j}$. If $\beta \in m_{n} \backslash\left(\bigcup_{j=0}^{k} G_{j}\right)$ then

$$
\beta \in \mathrm{Cl}\left(\bigcup_{j=1}^{k} F_{j}\right) \cap\left(\bigcap_{j=1}^{k} \mathrm{Cl}\left(D^{n} \backslash E_{j}\right)\right) .
$$

$\beta \in \mathrm{Cl}\left(\bigcup_{j=1}^{k} F_{j}\right)=\bigcup_{j=1}^{k} \mathrm{Cl}\left(F_{j}\right)$ implies $\beta \in \mathrm{Cl} F_{j}$ for some fixed $j$, say $j=$ 1 ; consequently, $|f(\beta)| \leqslant r$ for all $f \in F_{1}$. This implies the existence of a neighborhood $V$ of $\beta$ in $m_{n}$ on which $|f|<(r+t) / 2$ for all $f \in F_{1}$. We also have $\beta \in \mathrm{Cl}\left(D^{n} \backslash E_{1}\right)$ which implies $V \cap\left(D^{n} \backslash E_{1}\right)$ is not empty. But, $z \in D^{n} \backslash E_{1}$ implies there is some $f \in F_{1}$ such that $|f(z)| \geqslant t$, so $z \in V \cap$ $\left(D^{n} \backslash E_{1}\right)$ implies $t \leqslant|f(z)|<(r+t) / 2<t$ which is impossible. Therefore, $\beta$ cannot exist and $m_{n}=\bigcup_{j=0}^{k} G_{j}$.

5. Interpolating sequences in wedges. Our first example of an $\alpha$-separated sequences is given by the following proposition.

Proposition 5.1. If $S=\left\{a_{i}\right\}_{i=1}^{\infty} \subset D$ is a u.s. sequence and there exists $\tau \geqslant 0$ such that $S \subset\left\{z \in D:\left(1-|z|^{2}\right) /\left|1-z^{2}\right|>\tau\right\} \cap\{z \in D: \operatorname{Im}(z) \geqslant$ 0 \}. Then $S$ is $\alpha$-separated.

Proof. $S$ uniformly separated implies that

$$
\rho\left(a_{i}, a_{j}\right)=\left|\left(a_{i}-a_{j}\right) /\left(1-a_{i} \bar{a}_{j}\right)\right| \geqslant p \text { for some } p,
$$

and $\operatorname{Inf}_{i} \Pi_{j=1 ; j \neq i}^{\infty} \rho\left(a_{i}, a_{j}\right)>0$; so there exists $M$ such that $\Sigma_{j=1}^{\infty} 1-\rho\left(a_{i}, a_{j}\right) \leqslant$ $M$ for all $i$.

Let

$$
\alpha_{j}(z)=\frac{z-a_{j}}{1-z \bar{a}_{j}} \frac{z-\bar{a}_{j}}{1-z a_{j}}
$$

Then 


$$
\alpha_{j} \in H^{\infty}(D), \quad\left\|\alpha_{j}\right\| \leqslant 1, \quad \alpha_{j}\left(a_{j}\right)=0
$$

and

$$
\left|\alpha_{j}\left(a_{i}\right)\right|=\rho\left(a_{j}, a_{i}\right) \rho\left(\bar{a}_{j}, a_{i}\right) \geqslant p^{2} .
$$

(This last inequality follows from the fact that since $\operatorname{Im}\left(a_{i}\right) \geqslant 0$ and $\operatorname{Im}\left(a_{j}\right) \geqslant$ $0, \rho\left(\bar{a}_{j}, a_{i}\right) \geqslant \rho\left(a_{j}, a_{i}\right)$.) Standard arguments show that

$$
\sum_{j=1}^{\infty}\left|1-\alpha_{i}(z)\right|=\sum_{i=1}^{\infty} \frac{\left|\left(1-z^{2}\right)\right|\left(1-\left|a_{j}\right|^{2}\right)}{\left|\left(1-a_{j} z\right)\left(1-\bar{a}_{j} z\right)\right|}
$$

converges uniformly on compact disks in $D$.

Finally,

$$
\begin{aligned}
\left|1-\alpha_{j}\left(a_{i}\right)\right| & =\frac{\left|\left(1-a_{i}^{2}\right)\right|\left(1-\left|a_{j}\right|^{2}\right)}{\left|\left(1-a_{j} a_{i}\right)\left(1-\bar{a}_{j} a_{i}\right)\right|} \\
& =\frac{\left|1-a_{i}^{2}\right|}{1-\left|a_{i}\right|^{2}}\left|\frac{1-a_{j} \bar{a}_{i}}{1-a_{j} a_{i}}\right| \frac{\left(1-\left|a_{i}\right|^{2}\right)\left(1-\left|a_{j}\right|^{2}\right)}{\left|\left(1-a_{j} \bar{a}_{i}\right)\left(1-\bar{a}_{j} a_{i}\right)\right|} \\
& =\frac{\left|1-a_{i}^{2}\right|}{1-\left|a_{i}\right|^{2}}\left|\frac{1-a_{j} \bar{a}_{i}}{1-a_{j} a_{i}}\right|\left(1-\rho\left(a_{j} a_{i}\right)^{2}\right) \\
& \leqslant 2\left|\frac{1-a_{j} \bar{a}_{i}}{1-a_{j} a_{i}}\right| \frac{\left|1-a_{i}^{2}\right|}{1-\left|a_{i}\right|^{2}}\left(1-\rho\left(a_{j} a_{i}\right)\right) .
\end{aligned}
$$

A fairly direct calculation again shows that if $\operatorname{Im} a_{j} \geqslant 0$ and $\operatorname{Im} a_{i} \geqslant 0$ then $\left|\left(1-a_{j} \bar{a}_{i}\right) /\left(1-a_{j} a_{i}\right)\right| \leqslant 1$ so

$$
\sum_{j=1 ; j \neq i}^{\infty}\left|1-\alpha_{j}\left(a_{i}\right)\right| \leqslant 2 \frac{\left|1-a_{i}^{2}\right|}{1-\left|a_{i}\right|^{2}} \sum_{j=1 ; j \neq i}^{\infty}\left(1-\rho\left(a_{i}, a_{j}\right)\right) \leqslant \frac{2}{\tau} M .
$$

Therefore, $S$ is $\alpha$-separated.

COROllary 5.2. If $S=\left\{a_{i}\right\}_{i=1}^{\infty} \subset D$ is u.s. and there exists $\tau>0$ such that $S \subset\left\{z \in D:\left(1-|z|^{2}\right) /\left|1-z^{2}\right|>\tau\right\}$ then $S$ is a general interpolating sequence.

Proof. By Proposition 5.1, $S_{1}=S \cap\{z \in D: \operatorname{Im}(z) \geqslant 0\}$ and $S_{2}=$ $S \cap\{z \in D: \operatorname{Im}(z)<0\}$ are $\alpha$-separated and hence general interpolating sequences. But $S=S_{1} \cup S_{2}$ is u.s. in $D$ and consequently, by Carleson's theorem, $S$ is interpolating. Since $S_{1}$ and $S_{2}$ are disjoint subsequences of an interpolating sequence, $\mathrm{Cl}\left(S_{1}\right) \cap \mathrm{Cl}\left(S_{2}\right)=\varnothing$ and we can apply Corollary 4.4.

Our goal is to generalize Corollary 5.2 to a number of different contexts. We begin by considering the region

$$
W_{\tau}=\left\{z \in D:\left(1-|z|^{2}\right) /\left(\left|1-z^{2}\right|\right) \geqslant \tau\right\} .
$$


Corollary 5.2 tells us that if $S \subset W_{\tau}$ is a u.s. sequence then it is a general interpolating sequence. $W_{\tau}$ is readily seen to be the region in $D$ lying between the arcs of two circles which intersect at \pm 1 . (The circles have centers at $\pm i \tau \sqrt{1-\tau^{2}}$ and both have radius $1 / \sqrt{1-\tau^{2}}$.) In general, we make the following definition.

Definition 5.3. A wedge in $D$ is the region in $D$ lying between the arcs of two circles which intersect twice on the boundary of $D$.

Since linear fractional mappings send circles into circles, it is always possible to find a conformal self mapping of $D$ which maps any wedge into a subset of a wedge of the type $W_{\tau}=\left\{z \in D:\left(1-|z|^{2}\right) /\left|1-z^{2}\right| \geqslant \tau\right\}$. This fact immediately yields the following generalization of Corollary 5.2.

COROLlary 5.4. If $S \subset D$ is a u.s. sequence contained in a wedge, then $S$ is a general interpolating sequence.

Definition 5.5. We will call a region $R \subset D$ wedge-like if $R$ is contained in a finite union of wedges.

COROLlary 5.6. If $S \subset D$ is a u.s. sequence contained in a wedge-like region of $D$, then $S$ is a general interpolating sequence.

Proof. Since $S$ is contained in a wedge-like region, it has only a finite number of limit points (in the usual topology for $\mathrm{C}$ ) on $T^{\mathbf{1}}$. Call these limit points $\phi_{1}, \cdots, \phi_{k}$. It is clear that there exist constants $0<s<t<1$ such that for $j=1,2, \cdots, k, S \cap\left\{z \in D:\left|z-\phi_{j}\right|<t\right\}$ is contained in a wedge and is therefore a general interpolating sequence. By Lemma 4.6 there exist $G_{0}, G_{1}, \cdots, G_{k}$ open sets of $m_{1}$ such that $G_{0} \cap D=D\left(\bigcup_{j=1}^{k}\{z \in D\right.$ : $\left.\left.\left|z-\phi_{j}\right| \leqslant s\right\}\right)$ while $G_{j} \cap D=\left\{z \in D:\left|z-\phi_{j}\right|<t\right\}$ and $m_{1}=\bigcup_{j=0}^{k} G_{j}$. But $S \cap G_{0}$ will be a finite number of points hence general interpolating, and as we remarked above $S \cap G_{j}$ will be general interpolating for $j=1,2, \cdots, k$. The corollary then follows from Proposition 4.5.

We can generalize the above results to polydisks with the help of the constructive techniques of $\S \S 3$ and 4 .

Definition 5.7. A wedge $W$ in $D^{n}$ is the Cartesian product of $n$ wedges in $D$.

If $W_{\tau}=\left\{z \in D:\left(1-\left|z^{2}\right|\right) /\left(\left|1-z^{2}\right|\right) \geqslant \tau\right\}$ then it is easy to see that after application of a coordinate-wise conformal self-mapping of $D^{n}$ onto itself, any wedge in $D^{n}$ can be considered a subset of $W_{\tau} \times W_{\tau} \times \cdots \times W_{\tau}=W_{\tau}^{n}$ for an appropriate $\tau$.

Definition 5.8. A subset $\Omega \subset D^{n}$ is a near-wedge if there exists a wedge $W \subset D^{n-1}$ such that $\Omega=D \times W$. (If $n=1$, for consistency, we will say that a near-wedge is just all of $D$.)

The generalization we are after is: 
THEOREM 5.9. Suppose $S \subset D^{n}$ is a uniformly separated sequence, then

(a) if $S$ is contained in a wedge in $D^{n}$, then $S$ is a general interpolating sequence,

(b) if $S$ is contained in a near-wedge in $D^{n}, S$ is an (ordinary) interpolating sequence.

(Note. If $n=1$, Theorem 5.9 is just Carleson's theorem and Corollary 5.4.)

Proof. Assume $m$ is a u.s. bound for $S$. By virtue of the remarks following the definition of a wedge in $D^{n}$, we may assume all wedges are of the form, $W_{\tau} \times W_{\tau} \times \cdots \times W_{\tau}$. The proof progresses through a series of lemmas. If $S$ is a u.s. sequence in $D^{n}$ and $0<\epsilon<1$, define the $\epsilon$-neighborhood of $S$ as

$$
U(S, \epsilon)=\left\{z \in D^{n}: \rho_{n}(z, a)<\epsilon \text { for some } a \in S\right\} .
$$

$U(S, \epsilon)$ is the union of an infinite sequence of small polydisks. Since $S$ is u.s., if we always assume $\epsilon<1 / 2 m$, where $m$ is a u.s. bound for $S$, then it is clear that the polydisks of $U(S, \epsilon)$ will all be disjoint.

Lemma 5.10. For a given wedge $W \subset D^{n}$ there exist constants $\delta, \epsilon_{0}$, and $M_{0}>0$ such that for any $\epsilon<\epsilon_{0}$ there exist general interpolating sequences $S_{1}, S_{2}, \cdots, S_{N}$ where $N$ depends only on $\epsilon$, such that each $S_{i}$ has general interpolating bound $M_{0}$, and the $\epsilon$-neighborhoods of $S_{1}, S_{2}, \cdots, S_{N}$ cover $W$ (i.e. $W \subset \bigcup_{i=1}^{N} U\left(S_{i}, \epsilon\right)$ ). Moreover, each $S_{i}$ is of the form $S_{i}=R_{1} \times$ $R_{2} \times \cdots \times R_{n}$ where each $R_{j}$ is u.s. in $D$ with u.s. bound $1 / \delta$.

Proof. We begin by considering $W_{\tau}=\left\{z \in D:\left(1-\left|z^{2}\right|\right) /\left(\left|1-z^{2}\right|\right) \geqslant \tau\right\}$. Fix $\alpha, 3 / 4<\alpha<1$, say $\alpha=4 / 5$, and consider $\sigma: D \rightarrow D$ by $\sigma(z)=$ $(z+\alpha) /(1+\alpha z)$. $\sigma$ generates an infinite cyclic group of automorphism of $D$ with composition as the group operation $\left(\sigma^{-1}(z)=z-\alpha / 1-\alpha z\right)$. It is easy to verify that $\sigma\left(W_{\tau}\right)=W_{\tau}$, so $\sigma^{k}\left(W_{\tau}\right)=W_{\tau}$ for $k= \pm 1, \pm 2, \cdots$. It is also clear that $\sigma^{k}$ leaves no point of $W_{\tau}$ fixed for $k= \pm 1, \pm 2, \cdots$. Let $\gamma_{0}=$ $\left\{z \in W_{\tau}: \operatorname{Re} z=0\right\}$ and $\gamma_{1}=\sigma\left(\gamma_{0}\right)$ and let $W_{0}$ be the region of $W_{\tau}$ bounded on the left by $\gamma_{0}$ and on the right by $\gamma_{1}$. We will assume $W_{0}$ contains $\gamma_{0}$ but not $\gamma_{1}$. Define $W_{k}=\sigma^{k}\left(W_{0}\right), k= \pm 1, \pm 2, \cdots$. It is easy to see that $\left\{W_{k}\right\}_{k=-\infty}^{\infty}$ is a collection of disjoint subsets of $W_{\tau}$ and $W_{\tau}=\bigcup_{k=-\infty}^{\infty} W_{k}$.

Now suppose $z \in W_{0}$, let $z_{0}=z$ and $z_{k}=\sigma^{k}(z) \in W_{k}$ for $k=$ $\pm 1, \pm 2, \cdots$. Then if $k>0$, one verifies that

$$
\begin{aligned}
\frac{1-\left|z_{k}\right|}{1-\left|z_{k-1}\right|} & \leqslant 2 \frac{1-\left|z_{k}\right|^{2}}{1-\left|z_{k-1}\right|^{2}}=2 \frac{1-\left|\sigma\left(z_{k-1}\right)\right|^{2}}{1-\left|z_{k-1}\right|^{2}} \\
& \leqslant 2\left(1-|\alpha|^{2}\right)=2(1-16 / 25)=18 / 25<1 .
\end{aligned}
$$

So $z_{0}, z_{1}, z_{2}, \cdots$ is an exponential sequence in $D$, hence it is u.s. with u.s. 
bound derivable from the ratios $\left(1-\left|z_{k}\right|\right) /\left(1-\left|z_{k-1}\right|\right) \leqslant 18 / 25$. Similarly, $z_{-1}, z_{-2}, \cdots$ is u.s. in $D$, and it follows that $R_{z}=\left\{\cdots z_{-2}, z_{-1}, z_{0}, z_{1}\right.$, $\left.z_{2}, \cdots\right\}$ will be u.s. with u.s. bound depending only on the choice of $\alpha=4 / 5$. Hence, there are fixed constants, $\delta, M$ such that any sequence of the form $R_{z}$ will be u.s. with bound $1 / \delta$ and consequently a general interpolating sequence with bound $M$ where $M$ depends only on $\delta$ and $\tau$.

Let $\epsilon_{0}$ be small enough so that for any u.s. sequences $Q \subset D$ with u.s. bound $1 / \delta$, the disks of $U\left(Q, \epsilon_{0}\right)$ will be disjoint. We repeat that $M, \delta, \epsilon_{0}$ depend only on $W_{\tau}$ and the choice of $\alpha=4 / 5$. Suppose $\epsilon<\epsilon_{0}$, then we can cover $W_{0}$ by a finite number, say $r$, pseudo-hyperbolic disks, $D_{1}, D_{2}, \cdots$, $D_{r}$ of $\rho$-radius $\epsilon$ with centers in $W_{0}$. Thus, $W_{0} \subset \bigcup_{i=1}^{r} D_{i}$ and consequently $W_{k} \subset \bigcup_{i=1}^{r} \sigma^{k}\left(D_{i}\right)$. If $a_{1}, a_{2}, \cdots, a_{r}$ are the respective centers of $D_{1}, D_{2}$, $\cdots, D_{r}$ we let $R_{i}=R_{a_{i}}=\left\{\sigma^{k}\left(a_{i}\right)\right\}_{k=-\infty}^{\infty}$. Then, the sequences $R_{1}, R_{2}, \cdots$, $R_{r}$ will be general interpolating sequences in $D$, all with bound $M$, and $\bigcup_{i=1}^{r} U\left(R_{i}, \epsilon\right) \supset W_{\tau}$.

Finally, if $S=R_{i_{1}} \times R_{i_{2}} \times \cdots \times R_{i_{n}}$ is the Cartesian product of any $n$ of the $R_{i}$ 's, $S$ will be a general interpolating sequence in $D^{n}$ with general interpolating bound $M_{0}=M^{n}$. There are exactly $N=r^{n}$ such sequences, call them $S_{1}, S_{2}, \cdots, S_{N}$. Clearly $W_{\tau} \times W_{\tau} \times \cdots \times W_{\tau} \subset \bigcup_{i=1}^{N} U\left(S_{i}, \epsilon\right)$. This completes the proof of Lemma 5.10.

LEmma 5.11. Suppose $T \subset D^{n}$ is a u.s. sequence of the form $T=R_{1} \times$ $R_{2} \times \cdots \times R_{n}$ where $R_{k} \subset D$ is a u.s. sequence with u.s. bound $1 / \delta$ for $k=1,2, \cdots, n$. Let $\eta<\delta / 2$, then if $\epsilon<1 / 2 \eta^{3}$, there exist $f_{1}, f_{2}, \cdots$, $f_{n} \in H^{\infty}\left(D^{n}\right)$ such that $U(T, \epsilon) \subset\left\{z \in D^{n}:\left|f_{i}(z)\right|<\eta^{3}, i=1,2, \cdots, n\right\} \subset$ $U(T, \eta)$.

Proof. Let $B_{k} \in H^{\infty}(D)$ be the Blaschke product with zeros precisely at $R_{k}$. Then it can be shown (see for example [9, Lemmas 4.2 and 4.3]) that if $\eta$ is chosen such that $\eta<\delta / 2$, then

$$
\left\{z \in D:\left|B_{k}(z)\right|<\eta^{3}\right\} \subset\left\{z \in D: \rho_{1}(z, a)<\eta \text { for some } a \in R_{i}\right\} .
$$

Letting $f_{k} \in H^{\infty}\left(D^{n}\right)$ be defined by $f_{k}\left(\left\langle z^{(1)}, \cdots, z^{(n)}\right\rangle\right)=B_{k}\left(z^{(k)}\right)$, the lemma follows immediately.

Lemma 5.12. Suppose $S$ is a u.s. sequence of u.s. bound $m, T$ is a general interpolating sequence of bound $M$, and suppose $\eta<\operatorname{Min}\{1 / 2 m, 1 / 4 M\}$ then $S \cap U(T, \eta)$ is a general interpolating sequence.

Proof. $U(T, \eta)$ consists of a disjoint union of polydisks of the form $D_{a}=\left\{z \in D^{n}: \rho(z, a)<\eta\right\}$ where $a \in T$. By the choice of $\eta<1 / 2 m$, there exists at most one element of $S$ in $D_{a}$, hence $S \cap U(T, \eta)$ can be obtained by jiggling each point of a subsequence of $T$ a (pseudo-hyperbolic) distance 
$\leqslant 1 / 4 M$. Therefore, by Theorem 3.4, $S \cap U(T, \eta)$ is a general interpolating sequence.

Proof of Theorem 5.9(a). Let $\epsilon_{0}, \delta, M_{0}$ be the constants of Lemma 5.10 which, we recall, depend only on $W$. Let $\eta<\operatorname{Min}\left\{1 \frac{1}{2} \delta, 1 / 2 m, 1 / 4 M_{0}, \epsilon_{0}\right\}$ and let $\epsilon \leqslant 1 / 2 \eta^{3}$. Then by Lemma 5.10 there exist $N$ sequences $T_{1}, T_{2}, \cdots$, $T_{N}$ (where $N$ depends only on $\epsilon$ ) such that $W \subset \bigcup_{i=1}^{N} U\left(T_{i}, \epsilon\right.$ ), each $T_{i}$ is a general interpolating sequence of bound $M_{0}$, and is the Cartesian product of $n$ u.s. sequences in $D$, each with u.s. bound $1 / \delta$.

By Lemma 5.11, for each $T_{i}$ there is a family, $F_{i}$, of $n$ functions in $H^{\infty}\left(D^{n}\right)$ such that

$$
U\left(T_{i}, \epsilon\right) \subset\left\{z \in D^{n}:|f(z)|<\eta^{3} \text { for all } f \in F_{i}\right\} \subset U\left(T_{i}, \eta\right) .
$$

Applying Lemma 4.6, there exists $G_{0}, G_{1}, G_{2}, \cdots, G_{N}$ open subsets of $m_{n}$ such that $m_{n}=\bigcup_{i=0}^{N} G_{i}$ and

$$
\begin{aligned}
G_{0} \cap D^{n} & =D^{n} \mid \bigcup_{i=1}^{N}\left\{z \in D^{n}:|f(z)| \leqslant \epsilon \text { for all } f \in F_{i}\right\} \\
& \subset D^{n} \mid \bigcup_{i=1}^{N} U\left(T_{i}, \epsilon\right) \subset D^{n} \backslash W
\end{aligned}
$$

while

$$
G_{i} \cap D^{n}=\left\{z \in D^{n}:|f(z)|<\eta^{3} \text { for all } f \in F_{i}\right\} \subset U\left(T_{i}, \eta\right)
$$

for all $i=1,2, \cdots, N$. Moreover,

$$
S \cap G_{0}=S \cap G_{0} \cap D^{n} \subset S \cap\left(D^{n} \backslash W\right)=\varnothing
$$

while

$$
S \cap G_{i}=S \cap G_{i} \cap D^{n} \subset S \cap U\left(T_{i}, \eta\right)
$$

which, by Lemma 5.12 and the choice of $\eta$ is a general interpolating sequence for $i=1,2, \cdots, N$. Therefore we can apply Proposition 4.5 to demonstrate that $S$ is a general interpolating sequence. This completes the proof of part (a) of Theorem 5.9.

The proof of part (b) requires much of the machinery set up for part (a), so to avoid confusing changes in notation, we assume $S \subset W \times D \subset D^{n+1}$ is a u.s. sequence in $D^{n+1}$ of u.s. bound $m$, where $W$ is the same wedge we used in part (a). Under these assumptions, the constants $\epsilon_{0}, M_{0}, \delta$ and the sequences of Lemma 5.10 and the constants and functions of Lemma 5.11 can be used again. We must, however, modify Lemma 5.12. Using the notation of $\S 3$, we recall that a u.s. sequence in $D$ with u.s. bound $1 / \delta$ is interpolating, with interpolating bound $1 / \kappa(\delta)$. (This is the Shapiro and Shields estimate [14] for Carleson's theorem.) 
LEMMA 5.13. Suppose $S \subset D^{n+1}$ is u.s. with u.s. bound $m$, and $T \subset$ $D^{n}$ is a general interpolating sequence with bound $M$. Let $\epsilon<(1 / 4 m M) k(1 / 4 m)$ and let $U^{\prime}(T, \epsilon)=U(T, \epsilon) \times D$. Then $S \cap U^{\prime}(T, \epsilon)$ is an interpolating sequence.

Proof. $U^{\prime}(T, \epsilon)$ consists of "narrow polydisks" of the form

$$
P_{a}^{\prime}=\left\{\langle z, w\rangle \in D^{n} \times D: \rho_{n}(z, a)<\epsilon\right\} \quad \text { for } a \in T .
$$

For each $a \in T, S \cap P_{a}^{\prime}$ satisfies the hypothesis of Corollary 3.6 and therefore $\pi_{n+1}\left(S \cap P_{a}^{\prime}\right)$ is u.s. in $D$ with u.s. bound $4 m$. So by the Shapiro and Shields estimate, $\pi_{n+1}\left(S \cap P_{a}^{\prime}\right)$ is interpolating in $D$ with bound $1 / \kappa(1 / 4 m)$. By the remarks following Proposition 4.1, since $T$ is a general interpolating sequence with bound $M_{0}, Q=\left\{a \times \pi_{n+1}\left(S \cap P_{a}^{\prime}\right): a \in T\right\}$ will be an interpolating sequence with interpolating bound $M / \kappa(1 / 4 m)$. If $s \in S \cap U^{\prime}(T, \epsilon)$, then $s \in$ $S \cap P_{a}^{\prime}$ for some $a \in T$, and

$$
\left\langle a, \pi_{n+1}(s)\right\rangle \in a \times \pi_{n+1}\left(S \cap P_{a}^{\prime}\right) \subset Q
$$

and

$$
\rho_{n+1}\left(s,\left\langle a, \pi_{n+1}(s)\right\rangle\right)<\epsilon<(1 / 4 M) \kappa(1 / 4 m) .
$$

Moreover, since $S$ is a u.s. sequence with bound $m,\left\langle a, \pi_{n+1}(s)\right\rangle$ will have to be the only point in $Q$ which is within a pseudo-hyperbolic distance $\epsilon<1 / 4 m$ of $s$. Therefore, the points of $S \cap U^{\prime}(T, \epsilon)$ can be obtained by jiggling a subsequence of the points of $Q$ a pseudo-hyperbolic distance less than $\epsilon<(1 / 4 M) \kappa(1 / 4 m)$, where $M / \kappa(1 / 4 m)$ is an interpolating bound for $Q$. Consequently, we can apply Theorem 3.3, and it follows that $S$ is an interpolating sequence. This proves Lemma 5.13.

Proof of Theorem 5.9(b). The rest of the proof of part (b) of Theorem 5.9 mimics the final part of the proof of part (a). We have $S \subset W \times D$ is a u.s sequence with u.s. bound $m$. Let $\epsilon_{0}, \delta, M_{0}$ be the constants of Lemma 5.10 , let $\eta<\operatorname{Min}\left\{\epsilon_{0}, 1 / 2 \delta,\left(1 / 4 m M_{0}\right) \kappa(1 / 4 m)\right\}$, and choose $\epsilon<1 / 2 \eta^{3}$. Then by Lemma 5.10 there exist $N$ sequences $T_{1}, T_{2}, \cdots, T_{N}$ such that $W \times$ $D \subset \bigcup_{i=1}^{N} U^{\prime}\left(T_{i}, \epsilon\right)$ where each $T_{i}$ is a general interpolating sequence, of bound $M_{0}$, and is equal to the Cartesian product of $n$ u.s. sequences in $D$, each with u.s. bound $1 / \delta$. By Lemma 5.11 , for each $T_{i}$, we have a family $F_{i}$ of $n$ functions in $H^{\infty}\left(D^{n+1}\right)$ such that

$$
U^{\prime}\left(T_{i}, \epsilon\right) \subset\left\{z \in D^{n+1}:|f(z)|<\eta^{3} \text { for all } f \in F_{i}\right\} \subset U^{\prime}\left(T_{i}, \eta\right) .
$$

Applying Lemma 4.6, we get $H_{0}, H_{1}, \cdots, H_{N}$ open subsets of $m_{n+1}$ such that $m_{n+1}=\bigcup_{i=0}^{N} H_{i}$ and 


$$
\begin{aligned}
H_{0} \cap D^{n+1} & =D^{n+1} \backslash \bigcup_{i=1}^{N}\left\{z \in D^{n+1}:|f(z)| \leqslant \epsilon \text { for all } f_{i} \in \dot{F_{i}}\right\} \\
& \subset D^{n+1} \backslash \bigcup_{i=1}^{N} U^{\prime}\left(T_{i}, \epsilon\right) \subset D^{n+1} \backslash(W \times D)
\end{aligned}
$$

and

$$
\begin{aligned}
H_{i} \cap D^{n+1} & =\left\{z \in D^{n+1}:|f(z)|<\eta^{3} \text { for all } f_{i} \in F_{i}\right\} \\
& \subset U^{\prime}\left(T_{i}, \eta\right) \text { for } i=1,2, \cdots, N .
\end{aligned}
$$

Moreover

$$
S \cap H_{0} \subset S \cap\left(D^{n+1} \backslash W \times D\right)=\varnothing
$$

while

$$
S \cap H_{i} \subset S \cap U^{\prime}\left(T_{i}, \eta\right)
$$

which, by Lemma 5.13 and the choice of $\eta$, is an interpolating sequence for $i=1,2, \cdots, N$. We can therefore apply Proposition 4.5 from which it follows that $S$ is an interpolating sequence. This completes the proof of Theorem 5.9.

We can extend the results of Theorem 5.9 in a number of ways.

DEFINITION 5.14. A region $V \subset D^{n}$ will be called wedge-like if it contained in a finite union of wedges in $D^{n}$. A region $V^{\prime} \subset D^{n}$ will be called near wedge-like if $V^{\prime}=D \times V$ where $V$ is a wedge-like region in $D^{n-1}$.

CoROLlary 5.15. Suppose $S$ is a u.s. sequence in $D^{n}$, then

(a) if $S$ is contained in a wedge-like region of $D^{n}$, it is a general interpolating sequence,

(b) if $S$ is contained in a near wedge-like region of $D^{n}$, it is an interpolating sequence (w.r.t. C).

Finally, we point out that a careful examination of the proof of part (a) of Theorem 5.9 reveals that the hypothesis that $S$ is u.s. was used only to show that $S \cap D_{a}$ contained at most one point, where $D_{a}$ was a polydisk of small pseudo-hyperbolic radius. Clearly, a much weaker hypothesis than uniform separation will suffice.

THEOREM 5.16. If $S \subset D^{n}$ is a sequence with the property that $\operatorname{Inf}\{\rho(s, t): s, t \in S, s \neq t\}>0$ and $S$ is continued in a wedge-like region in $D^{n}$, then $S$ is a general interpolating sequence.

(In 1971, Tse Kam-fook [15] pointed out that pointwise separation in the pseudo-hyperbolic metric is sufficient for interpolation (w.r.t. C) inside a wedge in $D$. Theorem 5.16 is a broad generalization of this observation.) 
6. Examples of $\alpha$-separated sequences. We give a few examples of sequences (primarily in $D$ ) which satisfy Definition 2.4 and are therefore general interpolating sequences. One of the examples will show that $\alpha$-separation is not necessary for general interpolation, at least for sequences in $D^{n}$ where $n>1$. When $n=1$, it is not clear whether there is a distinction between uniformly separated, $\alpha$-separated, and general interpolating sequences.

We first give a very strong condition under which sequences are $\alpha$-separated.

DEFinition 6.1. A sequence $\left\{a_{i}\right\}_{i=1}^{\infty} \subset D^{n}$ is $\Pi$-separated if there exist $f_{i} \in H^{\infty}\left(D^{n}\right),\left\|f_{i}\right\| \leqslant 1$ and

$$
f_{i}\left(a_{j}\right)=\left\{\begin{array}{ll}
0 & \text { if } i \neq j \\
\delta_{i}>0 & \text { if } i=j
\end{array} \text { and } \prod_{i=1}^{\infty} \delta_{i}=p>0 .\right.
$$

(This condition is suggested by a more restrictive condition which A. Dufresnoy showed was sufficient for interpolation w.r.t. C.)

Proposition 6.2. A II-separated sequence in $D^{n}$ is $\alpha$-separated.

Proof. Let $\left\{a_{i}\right\}_{i=1}^{\infty}$ be $\Pi$-separated and let $f_{i}, \delta_{i}, p$ be as in the definition.

Let

$$
g_{i}(z)=\left(\delta_{i}-f_{i}(z)\right) /\left(1-\delta_{i} f_{i}(z)\right) .
$$

Then $g_{i} \in H^{\infty}\left(D^{n}\right),\left\|g_{i}\right\| \leqslant 1, g_{i}\left(a_{i}\right)=0$ and $g_{i}\left(a_{j}\right)=\delta_{i} \geqslant p$ if $i \neq j$. Consequently

$$
\sum_{i=1}^{\infty}\left|1-g_{i}\left(a_{j}\right)\right|=1+\sum_{i=1 ; i \neq j}^{\infty}\left(1-\delta_{i}\right) \leqslant 1+\sum_{i=1}^{\infty}\left(1-\delta_{i}\right) \leqslant \ln \frac{1}{p}+1 .
$$

Now we can assume $\delta_{1} \neq 1$, and if $i \neq 1, f_{i}\left(a_{1}\right)=0$. This implies $\left|f_{i}(z)\right| \leqslant \rho\left(z, a_{i}\right)$, so for each compact polydisk $\operatorname{Cl}\left(D^{n}(r)\right) \subseteq D^{n}$ there is a constant $R_{r}=\operatorname{Max}\left\{\rho\left(z, a_{1}\right) \mid z \in \mathrm{Cl}\left(D^{n}(r)\right)\right\}$ so that $\left|f_{i}(z)\right| \leqslant R_{r}$. Consequently,

$$
\left|1-g_{i}(z)\right|=\left|\frac{1-\delta_{i} f_{i}(z)-\delta_{i}+f_{i}(z)}{1-\delta_{i} f_{i}(z)}\right|=\left(1-\delta_{i}\right) \frac{\left|1+f_{i}(z)\right|}{\left|1-\delta_{i} f_{i}(z)\right|}
$$

and $\left|1-g_{i}(z)\right| \leqslant\left(2 / 1-R_{r}\right)\left(1-\delta_{i}\right)$ for all $z$ in $\mathrm{Cl}\left(D^{n}(r)\right)$ and $i>1$. Therefore

$$
\sum_{i=1}^{\infty}\left|1-g_{i}(z)\right| \leqslant 2\left(1+\frac{1}{1-R_{r}} \sum_{i=2}^{\infty}\left(1-\delta_{i}\right)\right) \leqslant 2\left(1+\frac{1}{1-R_{r}} \ln \frac{1}{p}\right)
$$

for all $z \in \mathrm{Cl}\left(D^{n}(r)\right)$. Therefore the $g_{i}$ 's satisfy all the conditions necessary to make $\left\{a_{i}\right\}_{i=1}^{\infty} \alpha$-separated.

It should be noted that although one can construct $\Pi$-separated sequences, II-separation is a very strong condition since the pseudo-hyperbolic distances between points in the sequence must be rapidly increasing. In fact, the minimum 
pseudo-hyperbolic distance, $d_{i}$, between $a_{i}$ and any other point of the sequence, must tend to one at a rate which is at least fast enough to insure $\Pi_{i=1}^{\infty} d_{i}>0$. Many interpolating sequences in $D$ do not have such increasing distances between points. For example, even sequences tending exponentially to the boundary are not necessarily II-separated. Indeed, if $a_{n}=1-r^{n}$ where $0<r<1$, then $\rho\left(a_{n}, a_{n+1}\right)=\frac{\left(1-r^{n+1}\right)-\left(1-r^{n}\right)}{1-\left(1-r^{n+1}\right)\left(1-r^{n}\right)}=\frac{r^{n}-r^{n+1}}{r^{n}+r^{n+1}-r^{2 n+1}}=\frac{1-r}{1+r+r^{n+1}}$ so $\rho\left(a_{n}, a_{n+1}\right) \rightarrow(1-r) /(1+r)$ as $n \rightarrow \infty$.

We mentioned that the result of Corollary 5.16 in the case of sequences in $D$ which were interpolating (w.r.t. C) was obtained by Tse Kam-fook. His observation was based on an earlier result of D. Newman [12] which stated that if $S=\left\{a_{n}\right\}_{n=1}^{\infty} \subset D$ converges nontangentially to the point 1 and $\operatorname{Inf} \rho\left(a_{j}, a_{j}\right)>$ 0 , then a necessary and sufficient condition that $S$ be an interpolating sequence (and hence, as we have seen, a general interpolating sequence) is

The set $\left\{\left(1-a_{n}\right) /\left(1-a_{m}\right): a_{n}, a_{m} \in S, n \neq m\right\}$ does not have 1 as a limit point.

By $a_{n} \rightarrow 1$ nontangentially, we mean that $a_{n} \rightarrow 1$ inside a wedge. We say that $a_{n} \rightarrow 1$ tangentially if $\left\{a_{n}\right\}_{n=1}^{\infty}$ is contained in no single wedge, i.e.

$$
\operatorname{Inf}_{n} \frac{1-\left|a_{n}\right|^{2}}{\left|1-a_{n}^{2}\right|}=0
$$

We show that under somewhat stronger tangential behavior $(N)$ will be sufficient to guarantee general interpolation.

Proposition 6.3. If

$$
S=\left\{a_{n}\right\}_{n=1}^{\infty} \subset D \text { and } \sum_{n=1}^{\infty} \frac{1-\left|a_{n}\right|^{2}}{\left|1-a_{n}^{2}\right|}=M<\infty
$$

and $\left\{\left(1-a_{n}\right) /\left(1-a_{m}\right): a_{n}, a_{m} \in S ; n \neq m\right\}$ does not have 1 as a limit point, then $S$ is $\alpha$-separated and consequently $S$ is a general interpolating sequence.

Proof. Let

$$
r=\operatorname{Inf}\left\{\left|1-\left(1-a_{n}\right) /\left(1-a_{m}\right)\right|: a_{n}, a_{m} \in S, n \neq m\right\} ;
$$

then $r>0$. Let

$$
\alpha_{n}(z)=\frac{\overline{a_{n}}}{\left|a_{n}\right|} \frac{a_{n}-z}{1-\bar{a}_{n} z}
$$

If $n \neq m$, suppose $\left|\left(1-a_{n}\right) /\left(1-a_{m}\right)\right| \leqslant 1$; then 


$$
\begin{aligned}
\left|\alpha_{n}\left(a_{m}\right)\right| & =\left|\alpha_{m}\left(a_{n}\right)\right|=\left|\frac{a_{n}-a_{m}}{1-a_{n} \bar{a}_{m}}\right| \\
& =\left|\frac{\left(1-a_{m}\right)-\left(1-a_{n}\right)}{1-a_{m}}\right|\left|\frac{1-a_{m}}{\left(1-a_{m}\right)+a_{m}\left(1-\bar{a}_{n}\right)}\right| \\
& =\left|1-\frac{1-a_{n}}{1-a_{m}}\right|\left|\frac{1}{1+a_{m}\left(1-\bar{a}_{n}\right) /\left(1-a_{m}\right)}\right| \\
& \geqslant r \frac{1}{1+\left|\left(1-\bar{a}_{n}\right) /\left(1-a_{m}\right)\right|} \geqslant \frac{r}{2} .
\end{aligned}
$$

So condition (1) and (2) of the definition of $\alpha$-separation are met. The hypothesis guarantees the convergence of the Blaschke product with zeroes at $\left\{a_{n}\right\}_{n=1}^{\infty}$, so condition (4) is also met.

Finally, if $n \neq m, r \leqslant 1-\left(1-a_{m}\right) /\left(1-a_{n}\right)=\left(a_{m}-a_{n}\right) /\left(1-a_{n}\right)$, so

$$
\begin{aligned}
\left|1-\alpha_{n}\left(a_{m}\right)\right| & =\frac{1-\left|a_{n}\right|}{\left|1-\bar{a}_{n} a_{m}\right|}|| a_{n}\left|+\bar{a}_{n} a_{m}\right| \\
& \leqslant 2 \frac{1-\left|a_{n}\right|}{\left|1-a_{n}\right|}\left|\frac{1-a_{n}}{a_{m}-a_{n}}\right|\left|\frac{a_{m}-a_{n}}{1-\bar{a}_{n} a_{m}}\right| \\
& =\frac{2}{r} \frac{1-\left|a_{n}\right|^{2}}{\left|1-a_{n}^{2}\right|} \frac{\left|1+a_{n}\right|}{1+\left|a_{n}\right|} \leqslant \frac{2}{r} \frac{1-\left|a_{n}\right|^{2}}{\left|1-a_{n}^{2}\right|}
\end{aligned}
$$

so $\sum_{n=1 ; n \neq m}^{\infty}\left|1-\alpha_{n}\left(a_{m}\right)\right| \leqslant 2 M / r$ and condition (3) is met; hence $S$ is $\alpha$-separated.

An example of a sequence satisfying the hypothesis of Proposition 4.18 is given by the following "doubly exponential" sequence:

$$
\begin{array}{r}
S=\left\{a_{n}\right\}_{n=1}^{\infty} \text { where } a_{n} \in\left\{z: 1-|z|=r_{1}^{n}\right\} \cap\left\{z:|1-z|=r_{2}^{n}\right\} \\
\text { where } 0<r_{1}<r_{2}<1 .
\end{array}
$$

On the other hand, the sequence, $T=\left\{b_{n}\right\}_{n=1}^{\infty}$ where

$$
b_{n}=\left\{z: 1-|z|=1 / n^{4}\right\} \cap\{z:|1-z|=1 / n\} \cap\{z: \operatorname{Im} z \geqslant 0\}
$$

is an example of an $\alpha$-separated sequence which satisfies all the hypotheses of Proposition 6.3 except condition (N).

We note that Corollary 5.4 and Proposition 6.3 exhibit u.s. sequences in $D$ with extreme tangential and nontangential behavior as general interpolating sequences.

In 1956, A. G. Naftalevitch described a method of constructing u.s. sequences 
in $D$ [11]. He showed that if $\left\{a_{n}\right\}_{n=1}^{\infty}$ is a nondecreasing sequence of points in the interval $I=(0,1)$ such that $\sum_{n=1}^{\infty}\left(1-a_{n}\right)<\infty$ then one could construct a u.s. sequence, $\left\{\lambda_{n}\right\}_{n=1}^{\infty}$, with $\left|\lambda_{n}\right|=a_{n}$. Using a somewhat simpler argument, we obtain a similar, slightly weaker, result for $\alpha$-separated sequences.

THEOREM 6.4. Suppose $\left\{a_{n}\right\}_{n=1}^{\infty}$ is a nondecreasing sequence of points in $I=(0,1)$ and $\Sigma_{n=1}^{\infty}\left(1-a_{n}\right)^{p}<\infty$ for some $p<1$, then there exists an $\alpha$ separated sequence $\left\{\lambda_{n}\right\}_{n=1}^{\infty} \subset D$ such that $\left|\lambda_{n}\right|=a_{n}$.

Proof. We may as well assume $p>1 / 2$. For $k=1,2,3, \cdots$ let

$$
I_{k}=\left\{x \in I: 1-1 / 2^{k-1}<x \leqslant 1-1 / 2^{k}\right\} \text {. }
$$

By throwing out a finite number of points of $\left\{a_{n}\right\}_{n=1}^{\infty}$, we can assume the sequence $\left\{a_{n}\right\}_{n=1}^{\infty}$ is embedded in another nondecreasing sequence of points in I, $\left\{\beta_{n}\right\}_{n=1}^{\infty}$, where $\left\{\beta_{n}\right\}_{n=1}^{\infty}$ has the following properties:

(1) $\beta_{n} \geqslant 1-1 / 2^{N}$ for some fixed $N$,

(2) $\left(1 / 2^{k}\right)^{1-p} \leqslant \Sigma_{\beta_{i} \in I_{k}}\left(1-\beta_{i}\right)^{p}$ for $k=N, N+1, \cdots$,

(3) $\Sigma_{i=1}^{\infty}\left(1-\beta_{i}\right)^{p} \leqslant \frac{1}{2}$.

(To see how to do this, take $N$ such that $\sum_{n=N}^{\infty}\left(1 / 2^{k}\right)^{1-p}<1 / 8$, and throw out a finite number of points of $\left\{a_{n}\right\}$ so that $a_{n} \geqslant 1-(1 / 2)^{N}$ for all $n$ and $\Sigma_{n}\left(1-a_{n}\right)^{p} \leqslant 1 / 4$. Since $p \geqslant 1 / 2,\left(1 / 2^{k}\right)^{p} \leqslant\left(1 / 2^{k}\right)^{1-p}$; so for each $k, k=$ $N, N+1, \cdots$, there exists an integer $r_{k}$ such that

$$
\left(1 / 2^{k}\right)^{1-p} \leqslant r_{k}\left(1 / 2^{k}\right)^{p} \leqslant 2\left(1 / 2^{k}\right)^{1-p}
$$

Construct the sequence

$$
\begin{gathered}
\left\{\gamma_{j}\right\}_{j=1}^{\infty}=\{\underbrace{1-\frac{1}{2^{N}}, \cdots, 1-\frac{1}{2^{N}}}_{r_{N} \text { times }}, \cdots, \underbrace{1-\frac{1}{2^{k}}, \cdots, 1-\frac{1}{2^{k}}}_{r_{k} \text { times }}, \\
\underbrace{\left.1-\frac{1}{2^{k+1}}, \cdots, 1-\frac{1}{2^{k+1}}, \cdots\right\}}_{r_{k+1} \text { times }} ;
\end{gathered}
$$

then

$$
\left(\frac{1}{2^{k}}\right)^{1-p} \leqslant \sum_{\gamma_{j} \in I_{k}}\left(1-\gamma_{j}\right)^{p}=r_{k}\left(\frac{1}{2^{k}}\right)^{p} \leqslant 2\left(\frac{1}{2^{k}}\right)^{1-p}
$$

Let $\left\{\beta_{n}\right\}_{n=1}^{\infty}$ be the sequence obtained from the points of $\left\{\gamma_{j}\right\}_{j=1}^{\infty} \bigcup\left\{\alpha_{i}\right\}_{i=1}^{\infty}$ reordered so as to form a nondecreasing sequence. Then 


$$
\begin{aligned}
\sum_{n=1}^{\infty}\left(1-\beta_{n}\right)^{p} & =\sum_{n=1}^{\infty}\left(1-a_{n}\right)^{p}+\sum_{n=1}^{\infty}\left(1-\gamma_{n}\right)^{p} \\
& \leqslant \frac{1}{4}+2 \sum_{k=N}^{\infty}\left(\frac{1}{2^{k}}\right)^{p} \leqslant \frac{1}{2} .
\end{aligned}
$$

The other two properties of $\left\{\beta_{n}\right\}_{n=1}^{\infty}$ are easily verified.)

We construct a sequence $\left\{\lambda_{n}\right\}_{n=1}^{\infty} \subset D$ such that $\left\{\lambda_{n}\right\}_{n=1}^{\infty}$ is $\alpha$-separated and $\left|\lambda_{n}\right|=\beta_{n}$ for $n=1,2, \cdots$.

Let

$$
\sigma_{1}=\sum_{i=1}^{\infty}\left(1-\beta_{i}\right)^{p}, \quad \sigma_{2}=\sum_{i=2}^{\infty}\left(1-\beta_{i}\right)^{p}, \cdots, \quad \sigma_{n}=\sum_{i=n}^{\infty}\left(1-\beta_{i}\right)^{p}, \cdots
$$

and let $\lambda_{j} \in\left\{z \in D:|z|=\beta_{j}\right\} \cap\left\{z \in \Delta:|1-z|=\sigma_{j}\right\}$. (Note that $\sigma_{j} \geqslant$ $\left(1-\beta_{j}\right)^{p}>1-\beta_{j}$ so the above intersection is nonempty.)

If $k>m$,

$$
\left|\lambda_{k}-\lambda_{m}\right| \geqslant\left|1-\lambda_{k}\right|-\left|1-\lambda_{m}\right|=\sigma_{k}-\sigma_{m}=\sum_{n=m}^{k-1}\left(1-\beta_{n}\right)^{p}
$$

Similarly, if $k<m$, then $\left|\lambda_{k}-\lambda_{m}\right| \geqslant \sum_{n=k}^{m-1}\left(1-\beta_{n}\right)^{p}$.

We define $\alpha_{n} \in H^{\infty}(D)$ by

$$
\alpha_{n}(z)=\frac{\bar{\lambda}_{n}}{\left|\lambda_{n}\right|} \frac{\lambda_{n}-z}{1-\bar{\lambda}_{n} z}
$$

then $\left|\alpha_{n}(z)\right| \leqslant 1$ for all $z, \alpha_{n}\left(\lambda_{n}\right)=0$, and since $\Sigma_{n=1}^{\infty}\left(1-\left|\lambda_{n}\right|\right)<\infty$ the usual arguments show $\sum_{n=1}^{\infty}\left(1-\alpha_{n}(z)\right)$ converges uniformly on compact subsets of $D$.

We are interested in $\Sigma_{n=1}^{\infty}\left|1-\alpha_{n}\left(\lambda_{k}\right)\right|$. If $n \neq k$,

$$
\begin{aligned}
\left|1-\alpha_{n}\left(\lambda_{k}\right)\right| & =\frac{1-\left|\lambda_{k}\right|}{\left|1-\bar{\lambda}_{n} \lambda_{k}\right|}|| \lambda_{n}\left|+\bar{\lambda}_{n} \lambda_{k}\right| \\
& \leqslant 2 \frac{\left(1-\left|\lambda_{k}\right|\right)}{\left|1-\bar{\lambda}_{n} \lambda_{k}\right|}=2 \frac{\left(1-\beta_{n}\right)}{\left|\lambda_{n}-\lambda_{k}\right|}\left|\frac{\lambda_{n}-\lambda_{k}}{1-\bar{\lambda}_{n} \lambda_{k}}\right| \\
& \leqslant 2 \frac{1-\beta_{n}}{\left|\lambda_{n}-\lambda_{k}\right|} .
\end{aligned}
$$

Now fix $k$. We consider three separate possibilities.

Case 1. $n>k$, then 


$$
\begin{aligned}
\frac{1}{2}\left|1-\alpha_{n}\left(\lambda_{k}\right)\right| & \leqslant \frac{1-\beta_{n}}{\sum_{j=k}^{n-1}\left(1-\beta_{j}\right)^{p}} \leqslant \frac{1-\beta_{n}}{(n-k)\left(1-\beta_{n-1}\right)^{p}} \\
& \leqslant \frac{1-\beta_{n}}{(n-k)\left(1-\beta_{n}\right)^{p}}=\frac{\left(1-\beta_{n}\right)^{1-p}}{n-k} \\
& \leqslant \frac{\left(1-\beta_{n-k}\right)^{1-p}}{n-k} .
\end{aligned}
$$

So

$$
\sum_{n=k+1}^{\infty}\left|1-\alpha_{n}\left(\lambda_{k}\right)\right| \leqslant 2 \sum_{j=1}^{\infty} \frac{\left(1-\beta_{j}\right)^{1-p}}{j}
$$

but $\left(1-\beta_{j}\right)^{1-p}$ is $p /(1-p)$ summable $(p>1 / 2$ implies $p /(1-p)>1)$ and $1 / j$ is $q=p /(2 p-1)$ summable; so by Hölder's inequality,

$$
\begin{aligned}
\sum_{n=k+1}^{\infty}\left|1-\alpha_{n}\left(\lambda_{k}\right)\right| & \leqslant 2 \sum_{j=1}^{\infty} \frac{\left(1-\beta_{j}\right)^{1-p}}{j} \\
& \leqslant 2\left(\sum_{j=1}^{\infty}\left(1-\beta_{j}\right)^{p}\right)^{(1-p) / p}\left(\sum_{j=1}^{\infty}\left(\frac{1}{j}\right)^{q}\right)^{1 / q}=C_{1} .
\end{aligned}
$$
$1 / 2^{j-1}$.

Cases 2 and 3. $n<k$, assume $\left|\lambda_{k}\right|=\beta_{k} \in I_{j}$, i.e. $1 / 2^{j} \leqslant\left(1-\beta_{k}\right) \leqslant$

Case 2. $1-\left|\lambda_{n}\right|=\left(1-\beta_{n}\right) \leqslant 1 / 2^{j-2}$. Then $\left(1-\beta_{n}\right) \leqslant 4\left(1-\beta_{k}\right)$, and, therefore

$$
\begin{aligned}
\frac{1}{2}\left|1-\alpha_{n}\left(\lambda_{k}\right)\right| & \leqslant \frac{1-\left|\lambda_{n}\right|}{\left|\lambda_{n}-\lambda_{k}\right|} \leqslant \frac{1-\beta_{n}}{\sum_{i=n}^{k-1}\left(1-\beta_{i}\right)^{p}} \leqslant \frac{4\left(1-\beta_{k}\right)}{(k-n)\left(1-\beta_{k-1}\right)} \\
& \leqslant \frac{4\left(1-\beta_{k}\right)}{(k-n)\left(1-\beta_{k}\right)^{p}}=\frac{4\left(1-\beta_{k}\right)^{1-p}}{k-n} \leqslant \frac{4\left(1-\beta_{k-n}\right)^{1-p}}{k-n} .
\end{aligned}
$$

So

$$
\sum_{\left\{n: \beta_{n} \in I_{j-1} \cup I_{j} ; n<k\right\}}\left|1-\alpha_{n}\left(\lambda_{k}\right)\right| \leqslant 8 \sum_{i=1}^{\infty} \frac{\left(1-\beta_{i}\right)^{1-p}}{i} \leqslant 8 C_{1} .
$$

Case 3. $\left|\lambda_{n}\right|=\beta_{n} \in I_{N} \cup \cdots \cup I_{j-2}$, i.e. $\left(1-\beta_{n}\right)>1 / 2^{j-2}$.

$$
\begin{aligned}
\sum_{\left\{n: \beta_{n} \in I_{N} \cup \ldots \cup I_{j-2}\right\}} & \left|1-\alpha_{n}\left(\lambda_{k}\right)\right| \\
& =\sum_{\left\{n: \beta_{n} \in I_{N}\right\}}+\sum_{\left\{n: \beta_{n} \in I_{N+1}\right\}}+\cdots+\sum_{\left\{n: \beta_{n} \in I_{j-2}\right\}} \text {. }
\end{aligned}
$$


If $\beta_{n} \in I_{i}$,

$$
\frac{1}{2}\left|1-\alpha_{n}\left(\lambda_{k}\right)\right| \leqslant \frac{1-\beta_{n}}{\sum_{l=n}^{k-1}\left(1-\beta_{l}\right)^{p}} \leqslant \frac{1-\beta_{n}}{Q}
$$

where $Q=\Sigma_{\beta_{l} \in I_{i+1}}\left(1-\beta_{l}\right)^{p} \geqslant\left(1 / 2^{i+1}\right)^{1-p}$ (by the second property of the sequence $\left.\left\{\beta_{l}\right\}_{l=1}^{\infty}\right)$.

But $\beta_{n} \in I_{i}$ implies $\left(1-\beta_{n}\right) \leqslant 1 / 2^{i-1}$. So

$$
\begin{aligned}
\frac{1}{2}\left|1-\alpha_{n}\left(\lambda_{k}\right)\right| & \leqslant \frac{\left(1-\beta_{n}\right)^{1-p}}{\left(1 / 2^{i+1}\right)^{1-p}}\left(1-\beta_{n}\right)^{p} \\
& \leqslant \frac{\left(1 / 2^{i-1}\right)^{1-p}}{\left(1 / 2^{i+1}\right)^{1-p}}\left(1-\beta_{n}\right)^{p} \leqslant 4\left(1-\beta_{n}\right)^{p} .
\end{aligned}
$$

Hence

$$
\sum_{\beta_{n} \in I_{N} \cup \ldots \cup I_{j-2}}\left|1-\alpha_{n}\left(\lambda_{k}\right)\right| \leqslant 8 \sum_{r=1}^{\infty}\left(1-\beta_{n}\right)^{p} \leqslant 4
$$

Consequently $\Sigma_{n=1}^{\infty}\left|1-\alpha_{n}\left(\lambda_{k}\right)\right| \leqslant 4+8 C_{1}+1+C_{1}=9 C_{1}+5$.

The proof is complete except to point out that in case (1) we showed that if $n>k,\left|1-\alpha_{n}\left(\lambda_{k}\right)\right| \leqslant 1-\beta_{n} \leqslant 1 / 2$ hence $\left|\alpha_{n}\left(\lambda_{k}\right)\right| \geqslant 1 / 2$. Clearly, if $n<k$, $\left|\alpha_{n}\left(\lambda_{k}\right)\right|=\left|\alpha_{k}\left(\lambda_{n}\right)\right| \geqslant 1 / 2$, so property (2) of Definition 2.4 is satisfied.

REMARK. An application of Theorem 6.4 shows that general interpolating sequences in $D^{n}(n \geqslant 2)$ need not be $\alpha$-separated. Recall that in $D$, a sequence $S=$ $\left\{a_{i}\right\}_{i=1}^{\infty}$ is u.s. iff

$$
\operatorname{Inf}_{i} \prod_{i=1 ; j \neq i}^{\infty}\left|\frac{a_{i}-a_{j}}{1-a_{i} \bar{a}_{j}}\right|=\operatorname{Inf}_{i} \prod_{j=1 ; j \neq i}^{\infty} \rho\left(a_{i}, a_{j}\right)>0
$$

Since the metric, $\rho$, is defined in $D^{n}$, we can define a sequence $S=\left\{a_{i}\right\}_{i=1}^{\infty}$ in $D^{n}$ to be "product uniformly separated" (p.u.s.) if $\operatorname{Inf}_{i} \Pi_{j=1 ; j \neq i}^{\infty} \rho\left(a_{i}, a_{j}\right)>0$. If $S \subset D^{n}$ is $\alpha$-separated (with $\alpha_{1}, \alpha_{2}, \alpha_{3}, \cdots$ the functions of Definition 2.4), then $\rho\left(a_{i}, a_{j}\right)>\left|\alpha_{i}\left(a_{i}\right)\right|$, and the remarks following Definition 2.4 guarantee $\operatorname{Inf}_{i} \Pi_{j=1 ; j \neq i}^{\infty} \rho\left(a_{i}, a_{j}\right)>0$, so $S$ is p.u.s.

Since p.u.s. is equivalent to u.s. and hence interpolation in $D$, one might ask whether u.s. sequences in $D^{n}$ are p.u.s. and whether p.u.s. sequences in $D^{n}$ are interpolating (w.r.t. C). We use Theorem 6.4 to give an example of a general interpolating (hence u.s.) sequence in $D^{2}$ which is not p.u.s. Since the sequence is not p.u.s. it is not $\alpha$-separated, so $\alpha$-separation is sufficient but not necessary for general interpolation in $D^{n}$ for $n \geqslant 2$.

Let $S_{1}=\left\{\lambda_{n}\right\} \subset D$ be the $\alpha$-separated sequence constructed by Theorem 6.4 with the property that $\left|\lambda_{n}\right|=1-1 / n^{2}$. Then $S_{1}$ is a general interpolating 
sequence and by Proposition 4.1, $S=S_{1} \times S_{1} \subset D^{2}$ is also a general interpolating sequence (and consequently interpolating and u.s.). We show that $S$ is not p.u.s.

Noting that $\lambda_{1}=0$, consider

$$
\begin{aligned}
\sum_{i, j=1}^{N}\left(1-\rho_{2}\left(\langle 0,0\rangle,\left\langle\lambda_{i}, \lambda_{j}\right\rangle\right)\right) \geqslant \sum_{i=2}^{N} \sum_{j=1}^{i}\left(1-\rho_{2}\left(\langle 0,0\rangle,\left\langle\lambda_{i}, \lambda_{j}\right\rangle\right)\right) \\
=\sum_{i=2}^{N} \sum_{j=1}^{i}\left(1-\rho_{1}\left(0, \lambda_{i}\right)\right)=\sum_{i=2}^{N} \sum_{j=1}^{i}\left(1-\left|\lambda_{i}\right|\right) \\
=\sum_{i=2}^{N} \frac{i}{i^{2}}=\sum_{i=2}^{N} \frac{1}{i} .
\end{aligned}
$$

Consequently, the sum $\Sigma_{\left\langle\lambda_{i}, \lambda_{j}\right\rangle \in S}\left(1-\rho_{2}\left(\langle 0,0\rangle,\left\langle\lambda_{i}, \lambda_{j}\right\rangle\right)\right)$ diverges, hence

$$
\prod_{\lambda=\left\langle\lambda_{i}, \lambda_{j}\right\rangle \in S ; \lambda \neq 0} \rho_{2}(\lambda, 0)=0,
$$

and $S$ is not p.u.s.

7. Other contexts. In this section we briefly outline applications of the above results to contexts other than that of bounded analytic functions on polydisks. First we simply replace $D^{n}$ with a multiply connected region of $\mathbf{C}$, then we replace the uniform algebras $H^{\infty}\left(D^{n}\right)$ by an arbitrary uniform algebra $A$.

Let $\Omega$ be an $n$-connected region in $\mathbf{C}$, bordered by $n$ simple closed curves $C_{1}, C_{2}, \cdots, C_{n}$. Let $\Omega_{1}, \Omega_{2}, \cdots, \Omega_{n}$ be the complements in the extended plane of the closures of the components of the complement of $\Omega$ with $\partial \Omega_{k}=C_{k}$. Denote by $H^{\infty}(\Omega), H^{\infty}(\Omega, A), H^{\infty}\left(\Omega_{k}\right)$, and $H^{\infty}\left(\Omega_{k}, A\right)$ the algebras of bounded analytic $C$ and $A$ valued functions defined on $\Omega$ and $\Omega_{k}$. We can define interpolating, general interpolating and uniformly separated sequences in $\Omega$ in a natural manner.

Let $\phi_{k}: \Omega_{k} \rightarrow D$ conformally with $\phi_{k}(z) \rightarrow T^{1}$ as $z \rightarrow C_{k}$ (recall $\left.T^{1}=\{z \in \mathbf{C}:|z|=1\}\right)$ and define $\phi: \Omega \rightarrow D^{n}$ by $\phi(z)=\left\langle\phi_{1}(z), \phi_{2}(z), \cdots, \phi_{n}(z)\right\rangle$.

DEFINITION 7.1. We say $W \subset \Omega$ is a wedge in $\Omega$ if $\phi(W)$ is contained in a wedge in $D^{n}$.

THEOREM 7.2. Suppose $S=\left\{a_{i}\right\}_{i=1}^{\infty} \subset \Omega$ is u.s., then

(a) $S$ is an interpolating sequence,

(b) if $S$ is contained in a wedge in $\Omega, S$ is a general interpolating sequence.

Proof. First we show that $\phi(S)$ is u.s. in $D^{n}$.

It is well known that there exists a constant $P$ depending only on $\Omega$ such 
that $f \in H^{\infty}(\Omega)$ and $\|f\| \leqslant 1$ implies the existence of $f_{1}, f_{2}, \cdots, f_{n} ; f_{k} \in$ $H^{\infty}\left(\Omega_{k}\right)$ and $\left\|f_{k}\right\| \leqslant P$ such that for $z \in \Omega, f(z)=\sum_{k=1}^{n} f_{k}(z)$.

If $f_{j} \in H^{\infty}(\Omega)$ and $\left\|f_{j}\right\| \leqslant 1$ and $f_{j}\left(a_{j}\right)=1 / M$ and $f_{j}\left(a_{i}\right)=0$ if $i \neq j$, then $f_{j}=\Sigma_{k=1}^{n} f_{j_{k}}, f_{j_{k}} \in H^{\infty}\left(\Omega_{k}\right),\left\|f_{j_{k}}\right\| \leqslant P$. Define $F_{j} \in H^{\infty}\left(D^{n}\right)$ by

$$
F_{j}\left(\left\langle z^{(1)}, \cdots, z^{(n)}\right\rangle\right)=\sum_{k=1}^{n} f_{j_{k}}\left(\phi_{k}^{-1}\left(z^{(k)}\right)\right) .
$$

Then $\left\|F_{j}\right\| \leqslant n P$ and

$$
\begin{aligned}
F_{j}\left(\phi\left(a_{i}\right)\right) & =\sum_{k=1}^{n} f_{j_{k}}\left(\phi_{k}^{-1}\left(\phi_{k}\left(a_{i}\right)\right)\right) \\
& =\sum_{k=1}^{n} f_{j_{k}}\left(a_{i}\right)=f_{j}\left(a_{i}\right)= \begin{cases}1 / M & \text { if } i=j, \\
0 & \text { if } i \neq j ;\end{cases}
\end{aligned}
$$

so $\phi(S)$ is u.s. in $D^{n}$.

For case (b) this is sufficient, since $\phi(S)$ is uniformly separated and contained in a wedge, and Theorem 5.9 can be applied to show $\phi(S)$ is a general interpolating sequence in $D^{n}$. Since $\phi$ is an analytic map from $\Omega$ to $D^{n}, S$ will be a general interpolating sequence in $\Omega$.

For case (a) we note that $\left|\phi_{k}(z)\right| \rightarrow 1$ for some $k$ implies $\left|\phi_{j}(z)\right|$ will be bounded away from 1 for $j \neq k$. Therefore, if $S_{j}$ is a sequence in $\Omega$ with all its cluster points on $C_{j}$, and $S_{j}^{\prime}$ is another sequence in $\Omega$ with all its cluster points on $C_{1} \cup C_{2} \cup \cdots \cup C_{j-1} \cup C_{j+1} \cup \cdots \cup C_{n}$, then $\mathrm{Cl}\left(S_{j}\right) \cap \mathrm{Cl}\left(S_{j}^{\prime}\right)=$ $\varnothing$. Consequently, by using Corollary 4.4 , we can assume $S$ has all its cluster points on $C_{1}$. In that case, there will exist $\epsilon$, such that $\left|\phi_{j}(S)\right|<1-\epsilon$ for $j=2,3, \cdots, n$. So, if $0<\tau<\epsilon / 2, \phi(S) \subset D \times W_{\tau} \times W_{\tau} \times \cdots \times W_{\tau}$. Where $W_{\tau}=\left\{z \in D:\left(1-|z|^{2}\right) /\left|1-z^{2}\right| \geqslant \tau\right\}$. Thus, $\phi(S)$ is contained in a near-wedge in $D^{n}$, and by Theorem 5.9, is an interpolating sequence. As above, this implies $S$ is an interpolating sequence in $\Omega$.

DEFINITION 7.3. If $z, w \in \Omega$ define the pseudo-hyperbolic distance, $\rho_{\Omega}$, between $z$ and $w$ to be $\rho_{\Omega}(z, w)=\operatorname{Sup}\left\{|f(z)|: f \in H^{\infty}(\Omega),\|f\| \leqslant 1, f(w)=0\right\}$.

Corollary 7.4. Suppose $S=\left\{a_{i}\right\}_{i=1}^{\infty}$ is a sequence in $\Omega$ with the property that $\operatorname{Inf}_{i \neq j} \rho_{\Omega}\left(a_{i}, a_{j}\right)>0$. If $S$ is contained in a finite union of wedges in $\Omega$ then $S$ is a general interpolating sequence.

Proof. We note that there is a constant $M$, depending only on $\Omega$, such that for any $f \in H^{\infty}(\Omega)$, where $\|f\| \leqslant 1$, there exists $F \in H^{\infty}\left(D^{n}\right)$ such that $\|F\| \leqslant M$ and $F \circ \phi=f$. (The proof of this fact is exactly the same as the argument of Theorem 7.2 that the image under $\phi$ of a u.s. sequence in $\Omega$ is u.s. in $D^{n} ; M=n P$.) Consequently, for any $z, w, \rho_{n}(\phi(z), \phi(w)) \geqslant(1 / M) \rho_{\Omega}(z, w)$. 
Therefore, $\phi(S)$ satisfies the hypothesis of 5.16 and is a general interpolating sequence, from which it follows that $S$ is a general interpolating sequence in $\Omega$.

As a final generalization of the results of the first six sections, we note that $H^{\infty}\left(D^{n}\right)$ is just a uniform algebra and $D^{n}$ is a subset of its maximal ideal space, $m_{n}$.

Definition 7.5. If $\boldsymbol{A}$ is a uniform algebra with maximal ideal space $m_{A}$, a discrete sequence, $S=\left\{a_{i}\right\}_{i=1}^{\infty} \subset m_{A}$ is an interpolating sequence for $A$ if the map $T: A \rightarrow l^{\infty}$ given by $T(X)=\left\{X\left(a_{i}\right)\right\}_{i=1}^{\infty}$ is surjective. If $S$ is an interpolating sequence for $A$, we again will have a constant, $M$, with the property that if $\left\{\lambda_{i}\right\}_{i=1}^{\infty} \in l^{\infty}$ and $\operatorname{Sup}_{i}\left|\lambda_{i}\right| \leqslant 1$, then there will be $X \in A$ such that $\|X\| \leqslant M$ and $X\left(a_{i}\right)=\lambda_{i}$. $M$ will be called an interpolating bound for $S$.

We note that the functions in $A$ are all $\mathbf{C}$ valued, so we will be interested only in "simple" interpolating sequences.

Definition 7.6. A discrete sequence $S=\left\{a_{i}\right\}_{i=1}^{\infty} \subset m_{A}$ is uniformly separated for $A$ if there exists a constant $M$ and elements, $X_{1}, X_{2}, \cdots \in A$, such that for all $i,\left\|X_{i}\right\| \leqslant M, X_{i}\left(a_{i}\right)=1$, and $X_{i}\left(a_{j}\right)=0$ for $j \neq i$. $M$ is called a u.s. bound for $S$.

We noted that for $A=H^{\infty}\left(D^{n}\right)$ and $S \subset D^{n}$, we had no example of a u.s. sequence which is not interpolating. However, if $A(D)$ is the algebra of continuous functions on the closed unit disk, $\bar{D}$, which are analytic inside $D$, and $S$ is a sequence contained in $D$ which is u.s. for $H^{\infty}(D)$ and has a finite number of limit points in $\bar{D}$, then $S$ is u.s. for the algebra $A(D)$, but it is not an interpolating sequence for $A(D)$. The problem is that unlike in $H^{\infty}(D)$, there are no functions in $A$ which "adhere" to bounded sequences of functions. This difference suggests a minimal necessary condition that a sequence be interpolating:

Proposition 7.7. A necessary condition that a sequence $S \subset m_{A}$ be interpolating is the following weak adherence condition:

There exists a constant $M$ such that for any sequence of elements $\left\{X_{i}\right\}_{i=1}^{\infty}$ in the unit ball of $A$, there exists $X \in A$ with $\|X\| \leqslant M$ such that a subsequence of the $\left\{X_{i}\right\}_{i=1}^{\infty}$ converges pointwise on $S$ to $X$.

Proof. The proof follows from the definition of interpolating sequence and the following lemma.

Lemma 7.8. For $\left\{\lambda^{(k)}\right\}_{k=1}^{\infty}$ a bounded sequence of elements of $l^{\infty}$, there exists a subsequence which converges "pointwise" to an element in $l^{\infty}$; i.e. if $\lambda^{(k)}=\left\{\lambda_{i}^{(k)}\right\}_{i=1}^{\infty}$ then there exists an indexing set $k_{1}, k_{2}, \cdots$ and a sequence $\lambda=\left\{\lambda_{i}\right\}_{i=1}^{\infty} \in l^{\infty}$ such that $\lambda_{i}^{\left(k_{j}\right)} \rightarrow \lambda_{i}$ for each $i$. 
Proof. The proof follows from a standard diagonalization argument.

It is well known that the algebras $H^{\infty}(D)$ and $H^{\infty}\left(D^{n}\right)$ are sequentially compact with respect to pointwise convergence in $D$ (or $D^{n}$ ). This is, of course, a much stronger statement than assertion (W), indeed it implies that (W) holds for any subset of $D$ (or $D^{n}$ ) and in all cases $M=1$. On the other hand, as we have seen, condition (W) is not satisfied by sequences in $D$ for the algebra $A(D)$.

Lemma 3.2, Corollary 3.4, Theorem 4.3, Corollary 4.4, and Proposition 4.5 and their proofs all have direct and natural analogues for interpolating sequences in $m_{A}$. We do not know of any neat hypothesis (like that of Theorem 5.9, for example) under which u.s. implies interpolation. However, we do have a generalization of Theorem 2.5.

Definition 7.9. If $\boldsymbol{A}$ is a uniform algebra and $S=\left\{a_{n}\right\}_{n=1}^{\infty} \subset m_{A}$, then $S$ is $\alpha$ separated if there exist constants $M$ and $p>0$ and a sequence $\left\{\alpha_{n}\right\}_{n=1}^{\infty} \subset A$ such that

(1) $\left\|\alpha_{n}\right\| \leqslant 1$ for all $n$.

(2) $\alpha_{n}\left(a_{n}\right)=0$ but $\left|\alpha_{n}\left(a_{m}\right)\right|>p$ if $n \neq m$.

(3) $\sum_{n=1}^{\infty}\left|1-\alpha_{n}\left(a_{m}\right)\right| \leqslant M$ for all $m$.

(4) Condition (W) holds for $S$.

THEOREM 7.10. If $A$ is a uniform algebra and $S$ is an $\alpha$-separated sequence in $m_{A}$, then $S$ is an interpolating sequence for $A$.

Proof. $S \alpha$-separated implies condition (W) holds, so let $M_{W}$ be the constant associated with condition (W) for $S$. We first show that $S$ is u.s. For fixed $j$, consider

$$
\mathrm{x}_{i}^{N}=\prod_{n=1 ; n \neq j}^{N} \alpha_{n}
$$

$\mathrm{x}_{j}^{N} \in A,\left\|\mathrm{x}_{j}^{N}\right\| \leqslant 1$ and $\mathrm{x}_{j}^{N}\left(a_{n}\right)=0$ if $n \neq j$ and $1 \leqslant n \leqslant N$, while (3) of Definition 7.8 implies $\left|\mathrm{x}_{j}^{N}\left(a_{i}\right)\right|>e^{-M / p}$. Applying condition (W) to $\left\{\mathrm{x}_{j}^{N}\right\}_{N=j+1}^{\infty}$ we get $\mathbf{x}_{j} \in A,\left\|\mathbf{x}_{j}\right\| \leqslant M_{W}$, such that $\mathbf{x}_{j}\left(a_{n}\right)=0$ if $n \neq j, n=1,2, \cdots$ and $\left|\mathrm{x}_{j}\left(a_{j}\right)\right|>e^{-M / p}$. So $S$ is u.s. with bound $1 / \delta=M_{W} e^{M / p}$. Therefore we can define $\mathrm{f}_{1}, \mathrm{f}_{2}, \cdots \in A$ such that $\left\|\mathrm{f}_{j}\right\| \leqslant 1$ and $\mathrm{f}_{j}\left(a_{i}\right)=0$ if $i \neq j$, and $f_{i}\left(a_{i}\right)=0$.

The rest of the proof proceeds in a manner similar to the proof of Theorem 2.5 (see Theorem 2.2 of [10]) except that we have no guarantee that the infinite products involved will converge. Hence we must show that interpolations can take place on finite subsequences and that all these interpolations can be performed with functions of a given norm. An application of condition (W) then yields the final results. 


\section{BIBLIOGRAPHY}

1. W. G. Bade and P. C. Curtis, Jr., Embedding theorems for commutative Banach algebras, Pacific J. Math. 18 (1966), 391-409. MR 34 \#1878.

2. A. Browder, Introduction to function algebras, Benjamin, New York, 1969. MR 39 \#7431.

3. L. Carleson, An interpolation problem for bounded analytic functions, Amer. J. Math. 80 (1958), 921-930. MR 22 \#8129.

4. A. Dufresnoy, Sur les compacts d'interpolation du spectre d'une algèbre uniforme et la propriété d'extension linéaire bornée, C. R. Acad. Sci. Paris Sér. A-B 273 (1971), A568-A571. MR 45 \#9142.

5. N. Dunford and J. T. Schwartz, Linear operators. I: General theory, Pure and Appl. Math., vol. 7, Interscience, New York, 1958. MR 22 \#8302.

6. P. L. Duren, Theory of $H^{p}$ spaces, Pure and Appl. Math., vol. 38, Academic Press, New York, 1970. MR 42 \#3552.

7. T. Gamelin, Uniform algebras, Prentice-Hall, Englewood Cliffs, N.J., 1969.

8. I. Glicksberg, Measures orthogonal to algebras and sets of antisymmetry, Trans. Amer. Math. Soc. 105 (1962), 415-435. MR 30 \#4164.

9. K. Hoffman, Bounded analytic functions and Gleason parts, Ann. of Math. (2) 86 (1967), 74-111. MR 35 \#5945.

10. E. Kronstadt, General interpolating sequences in disks and polydisks, Bull. Amer. Math. Soc. 80 (1974), 132-137.

11. A. Naftalevič, On interpolation by functions of bounded characteristic, Učen. Zap. Vilnius. Gos. Univ. 5 (1956), 5-27. (Russian)

12. D. Newman, Interpolation in $H^{\infty}$, Trans. Amer. Math. Soc. 92 (1959), 501-507. MR 22 \#8130.

13. W. Rudin, Function theory in polydisks, Benjamin, New York, 1969. MR 41 \#501.

14. H. Shapiro and A. Shields, On some interpolation problems for analytic functions, Amer. J. Math. 83 (1961), 513-532. MR 24 \#A3280.

15. Tse Kam-fook, Nontangential interpolating sequences and interpolation by normal functions, Proc. Amer. Math. Soc. 29 (1971), 351-354. MR 43 \#537.

16. N. Th. Varopoulos, Sur la réunion de deux ensembles d'interpolation d'une algèbre uniforme, C. R. Acad. Sci. Paris Sér. A-B 272 (1971), A950-A952. MR 43 \#5312b.

DEPARTMENT OF MATHEMATICS, UNIVERSITY OF MICHIGAN, ANN ARBOR, MICHIGAN 48104. 